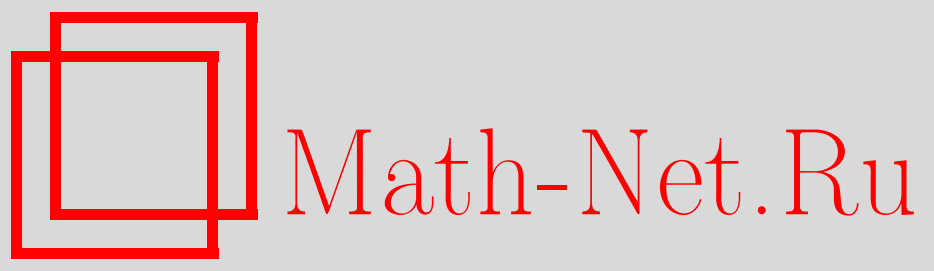

Д. Е. Александров, Об оценках мощности некоторых классов регулярных языков, Дискрет. матем., 2015, том 27, выпуск 2, 3-21

DOI: https://doi.org/10.4213/dm1322

Использование Общероссийского математического портала Math-Net.Ru подразумевает, что вы прочитали и согласны с пользовательским соглашением http://www . mathnet.ru/rus/agreement

Параметры загрузки:

IP : 54.224 .135 .184

26 апреля 2023 г., 11:01:29

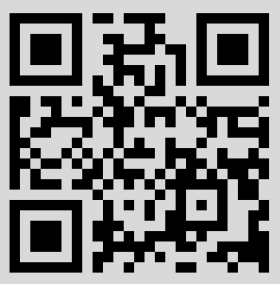




\title{
Об оценках мощности некоторых классов регулярных языков
}

\author{
๑ 2015 г. Д. Е. Александров*
}

\begin{abstract}
Рассмотрен метод модификации регулярных выражений для решения проблемы "экспоненциального взрыва" числа состояний конечного автомата, распознающего множество регулярных языков, задаваемых объединением регулярных выражений вида $* \mathrm{R}_{1} * \mathrm{R}_{2} *$, где $\mathrm{R}_{1}$ и $\mathrm{R}_{2}$ - произвольные регулярные выражения. Приведены оценки функций роста регулярных выражений из некоторого подкласса указанного класса выражений. Предложен способ оценки относительного роста числа слов регулярного языка, задаваемого парой регулярных выражений, при модификации этих выражений. Проанализирована практическая эффективность данного метода модификации выражений и предложенного способа оценки применительно к регулярным выражениям системы Snort.
\end{abstract}

Ключевые слова: конечные автоматы, регулярные выражения, системы обнаружения вторжений.

\section{1. Введение}

Одну из ключевых ролей в сфере информационных технологий играют экспертные системы, выносящие определенный вердикт относительно поданных на вход слов путем проверки принадлежности слова заранее заданному регулярному множеству. В частности, важное место в области информационной безопасности занимают сетевые системы обнаружения и (или) предотвращения вторжений, такие как Snort [1], Bro [2], L7-filter [3] и аппаратные продукты фирмы Cisco [4]. Все они имеют базы сигнатур - наборы регулярных выражений, задающие регулярные языки, слова которых признаются вредоносными.

Традиционно для проверки принадлежности слова заданному регулярному языку строится детерминированный конечный автомат (ДКА), принимающий данный язык. Однако с ростом числа выражений в наборе, задающем регулярный язык, возрастает пространственная сложность (число состояний) конечного автомата для проверки принадлежности слов заданному регулярному множеству.

Существует два основных подхода к преодолению данной трудности. Первый подход - модификация традиционных детерминированного и недетерминированного конечных автоматов. Так, например, в работе С. Кумара [5], в основе которой лежит алгоритм Ахо-Корасик [6], предлагается использовать ДКА с сокращенным определенным образом числом состояний. В работе [7] предлагается одновременно

*Место работы: МГУ им. М. В. Ломоносова, e-mail: dalexandrov@intsys.msu.ru 
использовать два специально сформированных автомата - ДКА и недетерминированный конечный автомат, благодаря которым объем требуемой памяти уменьшается по сравнению со случаем одного ДКА. В работах $[8,9]$ предлагается вводить специальные счетчики и битовые флаги, изменяемые в случае определенных переходов между состояниями, что также сокращает необходимый объем памяти.

Второй подход предполагает изменение исходного набора регулярных выражений таким образом, чтобы сократить сложность реализации в конечных автоматах за счет расширения определяемого выражениями регулярного языка. Однако методы, предлагаемые в работах по данной теме, как например в статье [10], подразумевают лишь "ручное" переписывание конкретных выражений. В работах $[11,12]$ предложен метод модификации произвольного набора выражений, принадлежащих классу выражений вида $* \mathrm{R}_{1} * \mathrm{R}_{2} *$ (в нотации PCRE [13]), где $\mathrm{R}_{1}$ и $\mathrm{R}_{2}$ - произвольные регулярные выражения, и даны оценки относительного выигрыша в числе состояний детерминированного конечного автомата для случая двух и более выражений.

В настоящей работе предложен способ оценки относительного роста числа слов распознаваемого регулярного языка при применении алгоритма статьи [11] к двум выражениям из класса $* \mathrm{R}_{1} * \mathrm{R}_{2} *$. Вначале кратко изложен метод расширения регулярного языка, заданного двумя выражениями. Далее описан метод оценки относительного роста числа слов при такой модификации. Затем приведены результаты применения этого алгоритма оценки к реально используемым выражениям.

\section{2. Основные понятия}

Пусть задан конечный алфавит $\Sigma$. Определим над подмножествами $M_{1}$ и $M_{2}$ множества всех слов над алфавитом $\Sigma$ операции конкатенации и “звезды Клини" следующим образом:

- $M_{1} M_{2}=\left\{\alpha_{1} \alpha_{2} \mid \alpha_{1} \in M_{1}, \alpha_{2} \in M_{2}\right\}$ - конкатенация множеств $M_{1}$ и $M_{2}$;

- $M_{1}^{*}=\{\Lambda\} \cup \bigcup_{i=1}^{\infty}\left\{\alpha_{j_{1}} \ldots \alpha_{j_{i}} \mid \alpha_{j_{k}} \in M_{1}, \forall k \in \overline{1, i}\right\}$ - звезда Клини множества $M_{1}$, где $\Lambda$ - пустое слово.

Тогда регулярное множество (язык) определяется рекурсивно $[14,15]$ :

- $\varnothing,\{\Lambda\}$ и $\{a\}-$ регулярные множества, где $a \in \Sigma$;

- если $M_{1}$ и $M_{2}$ - регулярные множества, то множества $M_{1} \cup M_{2}, M_{1} M_{2}$ и $M_{1}^{*}$ также являются регулярными множествами.

Для обозначения регулярных множеств используются регулярные выражения, традиционно определяющиеся следующим образом:

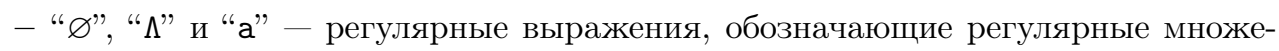
ства $\varnothing,\{\Lambda\}$ и $\{a\}$ соответственно, где $a \in \Sigma$;

- если $\mathrm{R}_{1}$ и $\mathrm{R}_{2}$ - регулярные выражения, обозначающие регулярные множества $M_{1}$ и $M_{2}$, то " $\mathrm{R}_{1}+\mathrm{R}_{2}$ " (объединение), " $\mathrm{R}_{1} \mathrm{R}_{2}$ " (конкатенация) и " $\mathrm{R}_{1}$ *" (звезда Клини) - регулярные выражения, обозначающие регулярные множества $M_{1} \cup M_{2}, M_{1} M_{2}$ и $M_{1}^{*}$ соответственно.

Легко проверить, что каждому выражению соответствует ровно одно регулярное множество, а каждому регулярному множеству соответствует не менее одного регулярного выражения.

В настоящей статье в регулярных выражениях через “.” будет обозначаться объединение всех символов алфавита, а через " $\left(\mathrm{R}_{1} \mid \mathrm{R}_{2}\right)$ " - объединение выражений 
$\mathrm{R}_{1}$ и $\mathrm{R}_{2}$. Отметим, что добавление обозначения “.” в регулярные выражения не расширяет класс регулярных языков. Следовательно, будет верна теорема Клини [15] о том, что множество слов является регулярным языком тогда и только тогда, когда существует инициальный детерминированный конечный автомат, распознающий его.

Пусть $\mathrm{R}$ - регулярное выражение над алфавитом $\Sigma, L(\mathrm{R})$ - регулярный язык, определяемый выражением $\mathrm{R}, V(L(\mathrm{R}))$ - приведенный ДКА, принимающий язык $L(\mathrm{R})$, а $|V|$ - число состояний автомата $V$. Через $L^{p f}(\mathrm{R})$ обозначим такое наибольшее подмножество $L(\mathrm{R})$, что ни одно из слов из $L(\mathrm{R})$ не является нетривиальным префиксом для слов языка $L^{p f}(\mathrm{R})$ (под нетривиальным префиксом подразумевается префикс, не совпадающий со всем словом). В случае, когда $L(\mathrm{R})$ содержит пустую строку $\Lambda$, положим $L^{p f}(\mathrm{R})=\{\Lambda\}$. Множество $L^{p f}(\mathrm{R})$ единственно и является регулярным языком [11].

\section{3. Модификация выражений}

В статьях $[11,12]$ была описана проблема экспоненциального роста числа состояний при объединении регулярных выражений вида $* \mathrm{R}_{1} * \mathrm{R}_{2} *$, а также предложен способ нивелирования данной проблемы. Пусть $\mathrm{R}^{1}=. * \mathrm{R}_{1} * \mathrm{R}_{2} *, \ldots, \mathrm{R}^{\mathrm{n}}=* * \mathrm{R}_{2 \mathrm{n}-1} \cdot * \mathrm{R}_{2 \mathrm{n}} * *-$ набор из $n$ регулярных выражений, где $n \geqslant 2$. Для сокращения числа состояний конечного автомата, распознающего принадлежность слова регулярному множеству $\bigcup_{i=1}^{n} L\left(\mathrm{R}^{\mathrm{i}}\right)$, предлагается расширить регулярное множество $\bigcup_{i=1}^{n} L\left(\mathrm{R}^{\mathrm{i}}\right)$ до множества $L\left(. *\left(\mathrm{R}_{1} \mid \mathrm{R}_{3}\right) . *\left(\mathrm{R}_{2} \mid \mathrm{R}_{4}\right) . *\right) \cup \bigcup_{i=3}^{n} L\left(\mathrm{R}^{\mathrm{i}}\right)$ и построить ДКА, распознающий его. И хотя новый автомат может ошибочно принимать слова не из языка $\bigcup_{i=1}^{n} L\left(\mathrm{R}^{\mathrm{i}}\right)$, он будет иметь не больше, а в некоторых случая заметно меньше состояний, чем автомат, принимающий язык $\bigcup_{i=1}^{n} L\left(\mathrm{R}^{\mathrm{i}}\right)$. В статьях $[11,12]$ приведены оценки чисел состояний исходных и новых автоматов. Далее будет предложен способ оценки относительного роста числа слов регулярного языка при таком расширении языка.

\section{4. Способ оценки}

Пусть $\mathrm{R}^{1}=. * \mathrm{R}_{1} * \mathrm{R}_{2} *$ и $\mathrm{R}^{2}=. * \mathrm{R}_{3} * \mathrm{R}_{4} * *$ - два регулярных выражения, причем, очевидно, языки $L\left(\mathrm{R}^{1}\right)=L\left(. * \mathrm{R}_{1} * \mathrm{R}_{2}\right) \Sigma^{*}$ и $L\left(\mathrm{R}^{2}\right)=L\left(. * \mathrm{R}_{3} * * \mathrm{R}_{4}\right) \Sigma^{*}$ бесконечны. Для сравнения бесконечных регулярных языков традиционно используют функцию роста - функцию вида $G_{l}(L)=|\{\alpha \in L|| \alpha \mid=l\}|$, где $l \in \mathbb{Z}_{+}, L \subseteq \Sigma^{*}$ - язык над алфавитом $\Sigma$. Тогда задача оценки роста регулярного языка при "слиянии" выражений сводится к оценке отношения

$$
\frac{G_{l}\left(L\left(. *\left(\mathrm{R}_{1} \mid \mathrm{R}_{3}\right) \cdot *\left(\mathrm{R}_{2} \mid \mathrm{R}_{4}\right) \cdot *\right)\right)}{G_{l}\left(L\left(\mathrm{R}^{1}\right) \cup L\left(\mathrm{R}^{2}\right)\right)}
$$

при различных $l \geqslant m=\min _{\alpha \in L\left(\mathrm{R}^{1}\right) \cup L\left(\mathrm{R}^{2}\right)}|\alpha|$. Однако вычисление отношения (1) для всякого $l$ из достаточно большого промежутка $[m, M]$ сопряжено с высокой сложностью нахождения функций роста для $M-m+1$ различных длин. Кроме того, если 
имеется $n$ различных выражений $\mathrm{R}^{\mathrm{i}}=. * \mathrm{R}_{2 \mathrm{i}-1} * \mathrm{R}_{2 \mathrm{i}} * *$, то для определения того, в каких случаях относительный прирост слов будет небольшим, необходимо вычислить отношение $(1)$ для $\frac{n \cdot(n-1)}{2}$ пар выражений.

В силу вышеописанных сложностей в случае подвыражений $\mathrm{R}_{\mathrm{i}}$ произвольного вида оценка роста регулярного языка при “слиянии" может быть трудоемкой. Для выделения класса регулярных подвыражений, имеющего практическую ценность, была проанализирована база сигнатур сетевой системы обнаружения вторжений Snort [16]. Было установлено, что подвыражения $\mathrm{R}^{\prime}$ и $\mathrm{R}^{\prime \prime}$ выражений $* * \mathrm{R}^{\prime} * \mathrm{R}^{\prime \prime} . *$, используемых в системе Snort, принадлежат классу выражений $R C$, состоящему из регулярных выражений вида $\mathrm{R}_{1} \mathrm{c}_{1} * \mathrm{R}_{2} \mathrm{c}_{2} * \ldots \mathrm{c}_{\mathrm{p}} * \mathrm{R}_{\mathrm{p}+1}$, где $p \geqslant 0$, языки $L\left(\mathrm{R}_{\mathrm{i}}\right)$ конечны, a $L\left(\mathrm{c}_{\mathrm{i}}\right) \subset \Sigma$.

Обозначим через $R F$ множество таких регулярных выражений $R$, что:

RF1) $L(\mathrm{R})$ не содержит пустых слов,

$\mathrm{RF} 2)$ при любом $\alpha \in L(\mathrm{R})$ никакое слово из $L(\mathrm{R})$, кроме $\alpha$, не содержит подслово $\alpha$, то есть $\Sigma^{*}\{\alpha\} \Sigma^{*} \cap L(\mathrm{R})=\{\alpha\}$,

RF3) при любых $\alpha, \beta \in L(\mathrm{R})$ никакой нетривиальный суффикс $\alpha$ не является префиксом $\beta$, то есть $\{\alpha\} \Sigma^{*} \cap \Sigma^{*}\{\beta\}=\{\alpha\} \Sigma^{*}\{\beta\}$.

Отметим, что среди 36 регулярных выражений системы Snort 22 выражения имеют подвыражения из класса $R F$.

Обозначим через $C_{l}(L)$ отношение $\frac{G_{l}(L)}{|\Sigma|^{l-m} \cdot G_{m}(L)}$, где $L-$ некоторый язык из $\Sigma^{*}$, a $m=\min _{\alpha \in L}|\alpha|$.

Пусть регулярное выражение $\mathrm{R}$ принадлежит классу $R C \cap R F$ и имеет вид $\mathrm{R}_{1} \mathrm{c}_{1} * \mathrm{R}_{2} \mathrm{c}_{2} * \ldots \mathrm{c}_{\mathrm{p}} * \mathrm{R}_{\mathrm{p}+1}$, тогда через $C^{\max }\left(L^{p f}(. * \mathrm{R})\right)$ обозначим произведение $\left(\prod_{i=1}^{p+1} \sum_{j=m_{i}}^{\infty} C_{j}\left(L\left(\mathrm{R}_{\mathrm{i}}\right)\right)\right) \cdot\left(\prod_{i=1}^{p} \frac{|\Sigma|}{|\Sigma|-k_{i}}\right)$, где $m_{i}=\min _{\alpha \in L\left(\mathrm{R}_{\mathrm{i}}\right)}|\alpha|, k_{i}=\left|L\left(\mathrm{c}_{\mathrm{i}}\right)\right|$.

Теорема 1. Пусть $. * \mathrm{R}_{1} * \mathrm{R}_{2} * u * \mathrm{R}_{3} * \mathrm{R}_{4} * *-$ mакие регулярные выражения, что подвыражения $\mathrm{R}_{\mathrm{i}}$ принадлежат классу $R C \cap R F, m_{1} \geqslant m_{3} u m_{2} \geqslant$ $\geqslant m_{4}$, где $m_{i}=\min _{\alpha \in L\left(\mathrm{R}_{\mathrm{i}}\right)}|\alpha|$. При любом $\epsilon \in(0,1)$ для всех таких длин $l$, что $m_{3}+m_{4} \leqslant l \leqslant \frac{\varepsilon}{\frac{G_{m_{i}}\left(L\left(\mathrm{R}_{\mathrm{i}}\right)\right)}{|\Sigma|^{m_{i}}} \cdot C^{\max }\left(L^{p f}\left(. * \mathrm{R}_{\mathrm{i}}\right)\right)}-1+2 m_{i}$ при $i \in\{3,4\}$, верно нера-

венство

$$
\begin{aligned}
& \frac{G_{l}\left(L\left(* *\left(\mathrm{R}_{1} \mid \mathrm{R}_{3}\right) \cdot *\left(\mathrm{R}_{2} \mid \mathrm{R}_{4}\right) \cdot *\right)\right)}{G_{l}\left(L\left(* \mathrm{R}_{1} \cdot * \mathrm{R}_{2} * *\right) \cup L\left(. * \mathrm{R}_{3} \cdot * \mathrm{R}_{4} \cdot *\right)\right)} \leqslant 1+ \\
& +\frac{1}{1-\varepsilon} \cdot\left(\frac{C^{\max }\left(L^{p f}\left(. * \mathrm{R}_{1}\right)\right) \cdot G_{m_{1}}\left(L\left(\mathrm{R}_{1}\right)\right)}{|\Sigma|^{m_{1}-m_{3}} \cdot G_{m_{3}}\left(L\left(\mathrm{R}_{3}\right)\right)}+\frac{C^{\max }\left(L^{p f}\left(* \mathrm{R}_{2}\right)\right) \cdot G_{m_{2}}\left(L\left(\mathrm{R}_{2}\right)\right)}{|\Sigma|^{m_{2}-m_{4}} \cdot G_{m_{4}}\left(L\left(\mathrm{R}_{4}\right)\right)}\right) .
\end{aligned}
$$

На практике (алфавит $\Sigma$ содержит 256 символов) значения произведений $\frac{G_{m_{i}}\left(L\left(\mathrm{R}_{\mathrm{i}}\right)\right)}{|\Sigma|^{m_{i}}} \cdot C^{\max }\left(L^{p f}\left(* \mathrm{R}_{\mathrm{i}}\right)\right)$ близки к $\frac{G_{m_{i}}\left(L\left(\mathrm{R}_{\mathrm{i}}\right)\right)}{|\Sigma|^{m_{i}}} \cdot \prod_{j=1}^{p} \frac{|\Sigma|}{|\Sigma|-k_{j}}$ и, в случае выражений системы Snort, в большинстве случаев имеют порядок $10^{-8}$ и меньше, то есть, например, при $\varepsilon=10^{-2}$ максимальная длина слов, на которых верна оценка, имеет порядок выше $10^{6}$. Верхняя же оценка относительного роста регулярного языка, предложенная в теореме 1, для пар выражений системы Snort в более чем трети случаев (при $\varepsilon=10^{-2}$ ) не превышает $10^{-2}$. 


\section{5. Вспомогательные утверждения}

Лемма 1. Пусть $\mathrm{R}$ - некоторое регулярное выражение. Тогда верно равенство

$$
G_{l}(L(* \mathrm{R} . *))=\sum_{i=m}^{l} G_{i}\left(L^{p f}(. * \mathrm{R})\right) \cdot|\Sigma|^{l-i},
$$

əде $m=\min _{\alpha \in L(\mathrm{R})}|\alpha|$.

Доказательство. Докажем индукцией по длине слов $l$. Очевидно, верно равенство $G_{m}(L(* \mathrm{R} . *))=G_{m}(L(\mathrm{R}))=G_{m}\left(L^{p f}(* \mathrm{R})\right)$.

Пусть $G_{n}(L(* \mathrm{R} . *))=\sum_{i=m}^{n} G_{i}\left(L^{p f}(. * \mathrm{R})\right) \cdot|\Sigma|^{n-i}$ при всех $n \leqslant l$. Докажем, что $L(. * \mathrm{R} . *) \cap \Sigma^{l+1}=\left(L(. * \mathrm{R} . *) \cap \Sigma^{l}\right) \Sigma \cup\left(L^{p f}(. * \mathrm{R}) \cap \Sigma^{l+1}\right) . \quad$ Множество $L(. * \mathrm{R} . *) \cap \Sigma^{l}$ состоит из всех слов длины $l$, содержащих подслова из $L(\mathrm{R})$. Тогда множество $\left(L(. * \mathrm{R} . *) \cap \Sigma^{l}\right) \Sigma$ содержит все слова длины $l+1$, у которых префиксы длины $l$ содержат подслова из $L(\mathrm{R})$. Множество $L^{p f}(. * \mathrm{R}) \cap \Sigma^{l+1}$ содержит все слова длины $l+1$, в которые подслова из $L(\mathrm{R})$ входят только в качестве суффиксов. Следовательно, множество $\left(L(. * \mathrm{R} . *) \cap \Sigma^{l}\right) \Sigma \cup\left(L^{p f}(. * \mathrm{R}) \cap \Sigma^{l+1}\right)$ содержит все слова длины $l+1$, содержащие подслова из языка $L(\mathrm{R})$, причем множества $\left(L(. * \mathrm{R} . *) \cap \Sigma^{l}\right) \Sigma$ и $L^{p f}(* \mathrm{R}) \cap \Sigma^{l+1}$ не пересекаются. Поэтому верно равенство

$$
\begin{gathered}
G_{l+1}(L(* \mathrm{R} . *))=\left|L(* \mathrm{R} . *) \cap \Sigma^{l+1}\right|= \\
=\left|\left(L(* \mathrm{R} . *) \cap \Sigma^{l}\right) \Sigma\right|+\left|L^{p f}(. * \mathrm{R}) \cap \Sigma^{l+1}\right|= \\
=G_{l}(L(* \mathrm{R} . *)) \cdots|\Sigma|+G_{l+1}\left(L^{p f}(. * \mathrm{R})\right)= \\
=\left(\sum_{i=m}^{l} G_{i}\left(L^{p f}(. * \mathrm{R})\right) \cdot|\Sigma|^{l-i}\right) \cdot|\Sigma|+G_{l+1}\left(L^{p f}(. * \mathrm{R})\right)= \\
=\sum_{i=m}^{l+1} G_{i}\left(L^{p f}(. * \mathrm{R})\right) \cdot|\Sigma|^{l+1-i} .
\end{gathered}
$$

Лемма 2. Пусть R - регулярное выражение из класса $R F$ u $m=\min _{\alpha \in L(\mathrm{R})}|\alpha|$. Тогда для всех $l \geqslant m$ верно равенство:

$$
\begin{aligned}
G_{l}\left(L^{p f}(* \mathrm{R})\right)=|\Sigma| \cdot G_{l-1}\left(L^{p f}(. * \mathrm{R})\right)+G_{l}(L(\mathrm{R})) & - \\
& -\sum_{i=m}^{l-m} G_{i}(L(\mathrm{R})) \cdot G_{l-i}\left(L^{p f}(. * \mathrm{R})\right) .
\end{aligned}
$$

Доказательство. Обозначим через $\bar{L}(\mathrm{R})$ множество слов из $\Sigma^{*}$, не содержащих подслова из $L(\mathrm{R})$, т. е. $\bar{L}(\mathrm{R})=\Sigma^{*} \backslash \Sigma^{*} L(\mathrm{R}) \Sigma^{*}$.

Докажем, что $L^{p f}(. * \mathrm{R})=\bar{L}(\mathrm{R}) L(\mathrm{R})$. Пусть $\alpha \in L^{p f}(. * \mathrm{R})$, тогда слово $\alpha$ представимо в виде $\alpha=\alpha_{1} \alpha_{2}$, где $\alpha_{1} \in \Sigma^{*}, \alpha_{2} \in L(\mathrm{R})$. Из определения $L^{p f}()$ следует, что ни один из префиксов слова $\alpha$, за исключением самого слова $\alpha$, не принадлежит языку $L(. * \mathrm{R})$. Следовательно, так как по определению $L(. * \mathrm{R})=\Sigma^{*} L(\mathrm{R})$, а слово $\alpha_{2}$ непусто, слово $\alpha_{1}$ не содержит подслова из $L(\mathrm{R})$, то есть $\alpha_{1} \in \bar{L}(\mathrm{R})$. Поэтому $L^{p f}(* \mathrm{R}) \subseteq \bar{L}(\mathrm{R}) L(\mathrm{R})$. 
Пусть $\alpha \in \bar{L}(\mathrm{R}) L(\mathrm{R})$, тогда слово $\alpha$ представимо в виде $\alpha=\alpha_{1} \alpha_{2}$, где $\alpha_{1} \in \bar{L}(\mathrm{R})$, $\alpha_{2} \in L(\mathrm{R})$. Докажем от противного, что $\alpha \in L^{p f}(* \mathrm{R})$. Пусть $\alpha \notin L^{p f}(. * \mathrm{R})$. Заметим, что $\alpha \in \bar{L}(\mathrm{R}) L(\mathrm{R}) \subseteq \Sigma^{*} L(\mathrm{R})=L(. * \mathrm{R})$, но тогда из определения $L^{p f}()$ и условия $\mathrm{RF} 1$ следует, что язык $L^{p f}(* \mathrm{R})$ содержит непустой префикс слова $\alpha$, не совпадающий со всем словом. Поэтому слово $\alpha$ также представимо в виде $\alpha=\beta_{1} \beta_{2}$, где $\beta_{1} \in$ $L^{p f}(. * \mathrm{R})$, а $\beta_{2}-$ некоторое непустое слово. Пусть $\left|\beta_{1}\right| \leqslant\left|\alpha_{1}\right|$, т. е. $\beta_{1}$ является префиксом слова $\alpha_{1}$. Так как $\beta_{1} \in L^{p f}(. * \mathrm{R})$, то $\beta_{1}$ содержит подслово из языка $L(\mathrm{R})$, а, следовательно, $\alpha_{1}$ также содержит подслово из $L(\mathrm{R})$. Но тогда $\alpha_{1} \notin \bar{L}(\mathrm{R})$. Противоречие.

Пусть $\left|\beta_{1}\right|>\left|\alpha_{1}\right|$. Так как $\beta_{1}$ принадлежит языку $L^{p f}(* \mathrm{R})$, то $\beta_{1}=\beta_{1}^{\prime} \beta_{1}^{\prime \prime}$, где $\beta_{1}^{\prime} \in \Sigma^{*}, \beta_{1}^{\prime \prime} \in L(\mathrm{R})$. Тогда, если $\left|\beta_{1}^{\prime}\right|<\left|\alpha_{1}\right|$, то нетривиальный суффикс слова $\beta_{1}^{\prime \prime} \in L(\mathrm{R})$ является префиксом слова $\alpha_{2} L(\mathrm{R})$. Противоречие с условием RF3.

Пусть $\left|\beta_{1}^{\prime}\right| \geqslant\left|\alpha_{1}\right|$, тогда слово $\alpha_{2}$ содержит слово $\beta_{1}^{\prime \prime}$, причем верно неравенство $\left|\beta_{1}^{\prime \prime}\right|<\left|\alpha_{2}\right|$, т. к. $\left|\beta_{2}\right|>0$. Но тогда множество $\Sigma^{*}\left\{\beta_{1}^{\prime \prime}\right\} \Sigma^{*} \cap L(\mathrm{R})$ содержит не только слово $\beta_{1}^{\prime \prime}$, но и слово $\alpha_{2} \neq \beta_{1}^{\prime \prime}$. Противоречие с условием RF2. Следовательно, $L^{p f}(* \mathrm{R})=\bar{L}(\mathrm{R}) L(\mathrm{R})$.

Вычислим функцию роста $G_{l}\left(L^{p f}(. * \mathrm{R})\right)=G_{l}(\bar{L}(\mathrm{R}) L(\mathrm{R}))$. Очевидно, что $(\bar{L}(\mathrm{R}) L(\mathrm{R})) \cap \Sigma^{l}=\bigcup_{i=0}^{l}\left(\bar{L}(\mathrm{R}) \cap \Sigma^{l-i}\right)\left(L(\mathrm{R}) \cap \Sigma^{i}\right)$. Докажем от противного, что множества $\left(\bar{L}(\mathrm{R}) \cap \Sigma^{l-i}\right)\left(L(\mathrm{R}) \cap \Sigma^{i}\right)$ и $\left(\bar{L}(\mathrm{R}) \cap \Sigma^{l-j}\right)\left(L(\mathrm{R}) \cap \Sigma^{j}\right)$ не пересекаются при $i \neq j$. Пусть пересечение $\left(\left(\bar{L}(\mathrm{R}) \cap \Sigma^{l-i}\right)\left(L(\mathrm{R}) \cap \Sigma^{i}\right)\right) \cap$ $\cap\left(\left(\bar{L}(\mathrm{R}) \cap \Sigma^{l-j}\right)\left(L(\mathrm{R}) \cap \Sigma^{j}\right)\right)$ содержит слово $\alpha$. Тогда $\alpha$ представимо в виде $\alpha=\alpha_{1} \alpha_{2}=\beta_{1} \beta_{2}$, где $\alpha_{1} \in \bar{L}(\mathrm{R}) \cap \Sigma^{l-i}, \alpha_{2} \in L(\mathrm{R}) \cap \Sigma^{i}, \beta_{1} \in \bar{L}(\mathrm{R}) \cap \Sigma^{l-j}$, $\beta_{2} \in L(\mathrm{R}) \cap \Sigma^{j}$. Без ограничения общности будем считать, что $i>j$. Тогда слово $\beta_{2}$ является суффиксом слова $\alpha_{2}$. Поэтому множество $\Sigma^{*}\left\{\alpha_{2}\right\} \Sigma^{*} \cap$ $\cap L(\mathrm{R})$ содержит слово $\beta_{2} \neq \alpha_{2}$. Противоречие с условием RF2. Следовательно, верно равенство

$$
\begin{gathered}
G_{l}(\bar{L}(\mathrm{R}) L(\mathrm{R}))=\left|\bigcup_{i=0}^{l}\left(\bar{L}(\mathrm{R}) \cap \Sigma^{l-i}\right)\left(L(\mathrm{R}) \cap \Sigma^{i}\right)\right|= \\
=\sum_{i=0}^{l}\left|\left(\bar{L}(\mathrm{R}) \cap \Sigma^{l-i}\right)\left(L(\mathrm{R}) \cap \Sigma^{i}\right)\right|=\sum_{i=0}^{l} G_{l-i}(\bar{L}(\mathrm{R})) \cdot G_{i}(L(\mathrm{R})),
\end{gathered}
$$

причем $G_{l}(\bar{L}(\mathrm{R}) L(\mathrm{R}))=\sum_{i=m}^{l} G_{l-i}(\bar{L}(\mathrm{R})) \cdot G_{i}(L(\mathrm{R}))$, так как $G_{i}(L(\mathrm{R}))=0$ при $0 \leqslant i<m$. Применив лемму 1 , получаем

$$
G_{l}(\bar{L}(\mathrm{R}))=|\Sigma|^{l}-G_{l}(L(. * \mathrm{R} . *))=|\Sigma|^{l}-\sum_{i=m}^{l} G_{i}\left(L^{p f}(. * \mathrm{R})\right) \cdot|\Sigma|^{l-i} .
$$


Поэтому

$$
\begin{gathered}
G_{l}\left(L^{p f}(* \mathrm{R})\right)=\sum_{i=0}^{l} G_{l-i}(\bar{L}(\mathrm{R})) \cdot G_{i}(L(\mathrm{R}))= \\
=\sum_{i=0}^{l} G_{i}(L(\mathrm{R})) \cdot\left(|\Sigma|^{l-i}-\sum_{j=m}^{l-i} G_{j}\left(L^{p f}(. * \mathrm{R})\right) \cdot|\Sigma|^{l-i-j}\right)= \\
=|\Sigma|^{l} \cdot \sum_{i=0}^{l} \frac{G_{i}(L(\mathrm{R}))}{|\Sigma|^{i}} \cdot\left(1-\sum_{j=m}^{l-i} \frac{G_{j}\left(L^{p f}(. * \mathrm{R})\right)}{|\Sigma|^{j}}\right) .
\end{gathered}
$$

Перепишем предыдущее равенство:

$$
\begin{gathered}
G_{l}\left(L^{p f}(. * \mathrm{R})\right)=|\Sigma| \cdot|\Sigma|^{l-1} \cdot\left(\sum_{i=0}^{l-1} \frac{G_{i}(L(\mathrm{R}))}{|\Sigma|^{i}} \cdot\left(1-\sum_{j=m}^{l-1-i} \frac{G_{j}\left(L^{p f}(* \mathrm{R})\right)}{|\Sigma|^{j}}\right)+\right. \\
\left.+\frac{G_{l}(L(\mathrm{R}))}{|\Sigma|^{l}} \cdot\left(1-\sum_{j=m}^{0} \frac{G_{j}\left(L^{p f}(. * \mathrm{R})\right)}{|\Sigma|^{j}}\right)-\sum_{i=m}^{l-1} \frac{G_{i}(L(\mathrm{R}))}{|\Sigma|^{i}} \cdot \frac{G_{l-i}\left(L^{p f}(* \mathrm{R})\right)}{|\Sigma|^{l-i}}\right)= \\
=|\Sigma| \cdot G_{l-1}\left(L^{p f}(. * \mathrm{R})\right)+G_{l}(L(\mathrm{R}))-\sum_{i=m}^{l-1} G_{i}(L(\mathrm{R})) \cdot G_{l-i}\left(L^{p f}(* \mathrm{R})\right) .
\end{gathered}
$$

В силу того, что $G_{l}\left(L^{p f}(. * \mathrm{R})\right)=0$ при $l<m$, верно доказываемое равенство:

$$
\begin{aligned}
G_{l}\left(L^{p f}(. * \mathrm{R})\right)=|\Sigma| \cdot G_{l-1}\left(L^{p f}(. * \mathrm{R})\right)+G_{l}(L(\mathrm{R})) & - \\
& -\sum_{i=m}^{l-m} G_{i}(L(\mathrm{R})) \cdot G_{l-i}\left(L^{p f}(. * \mathrm{R})\right) .
\end{aligned}
$$

Так как $\min _{\alpha \in L^{p f}(* \mathrm{R})}|\alpha|=\min _{\alpha \in L(\mathrm{R})}|\alpha|$, то равенство из леммы 2 можно переписать в следующем виде:

$$
\begin{gathered}
|\Sigma|^{l-m} \cdot G_{m}\left(L^{p f}(* \mathrm{R})\right) \cdot C_{l}\left(L^{p f}(* \mathrm{R})\right)= \\
=|\Sigma|^{l-m} \cdot G_{m}\left(L^{p f}(* \mathrm{R})\right) \cdot C_{l-1}\left(L^{p f}(* \mathrm{R})\right)+|\Sigma|^{l-m} \cdot G_{m}(L(\mathrm{R})) \cdot C_{l}(L(\mathrm{R})) \\
-\sum_{i=m}^{l-m}|\Sigma|^{i-m} \cdot G_{m}(L(\mathrm{R})) \cdot C_{i}(L(\mathrm{R})) \cdot|\Sigma|^{l-i} \cdot G_{m}\left(L^{p f}(* \mathrm{R})\right) \cdot C_{l-i}\left(L^{p f}(* \mathrm{R})\right) .
\end{gathered}
$$

Заметим, что $G_{m}\left(L^{p f}(. * \mathrm{R})\right)=G_{m}(L(\mathrm{R}))$, поэтому

$$
\begin{aligned}
C_{l}\left(L^{p f}(. * \mathrm{R})\right)=C_{l-1}\left(L^{p f}(* \mathrm{R})\right) & +C_{l}(L(\mathrm{R}))- \\
& -\frac{C_{m}(L(\mathrm{R}))}{|\Sigma|^{m}} \cdot \sum_{i=m}^{l-m} C_{i}(L(\mathrm{R})) \cdot C_{l-i}\left(L^{p f}(. * \mathrm{R})\right) .
\end{aligned}
$$

Лемма 3. Пусть регулярное выражение $\mathrm{R} \in R F$ задает регулярный язык $L(\mathrm{R})=\left\{\alpha_{1}, \ldots, \alpha_{n}\right\}$, где $\left|\alpha_{i}\right|=m$ при всех $i$. Тогда при $l \geqslant m$ верно равенство

$$
C_{l}\left(L^{p f}(. * \mathrm{R})\right)=1-\varepsilon(l),
$$

где $|\varepsilon(l)| \leqslant \frac{n \cdot(l+1-2 m)}{|\Sigma|^{m}}$ при $l \geqslant 2 m$ и $\varepsilon(l)=0$ в осталъных случаях. 
Доказательство. По условию $G_{m}(L(\mathrm{R}))=n, G_{i}(L(\mathrm{R}))=0$ при $i \neq m$. Тогда

$$
\begin{gathered}
C_{m}\left(L^{p f}(\mathrm{R})\right)=1, C_{l}\left(L^{p f}(\mathrm{R})\right)=C_{l-1}\left(L^{p f}(\mathrm{R})\right)=\ldots=1 \text { при } m<l<2 m, \\
C_{l}\left(L^{p f}(\mathrm{R})\right)=C_{l-1}\left(L^{p f}(\mathrm{R})\right)-\frac{n}{|\Sigma|^{m}} \cdot C_{l-m}\left(L^{p f}(. * \mathrm{R})\right) \text { при } l \geqslant 2 m .
\end{gathered}
$$

Заметим, что, так как $C_{l}\left(L^{p f}(. * \mathrm{R})\right) \geqslant 0$ при любых $l$, то функция $C_{l}\left(L^{p f}(. * \mathrm{R})\right)$ не возрастает по $l$ при $l \geqslant m$ и не превосходит $C_{m}\left(L^{p f}(. * \mathrm{R})\right)=1$. Следовательно, при $l \geqslant 2 m$ верно неравенство $C_{l}\left(L^{p f}(. * \mathrm{R})\right) \geqslant C_{l-1}\left(L^{p f}(. * \mathrm{R})\right)-\frac{n}{|\Sigma|^{m}}$. Поэтому при $l \geqslant 2 m$ верна также нижняя оценка $C_{l}\left(L^{p f}(. * \mathrm{R})\right) \geqslant C_{2 m-1}\left(L^{p f}(. * \mathrm{R})\right)-$ $\frac{n \cdot(l+1-2 m)}{|\Sigma|^{m}}$.

Таким образом при $l \geqslant 2 m$ верно неравенство

$$
C_{2 m-1}\left(L^{p f}(. * \mathrm{R})\right)-\frac{n \cdot(l+1-2 m)}{|\Sigma|^{m}} \leqslant C_{l}\left(L^{p f}(. * \mathrm{R})\right) \leqslant 1 .
$$

Лемма 4. Пусть регулярное выражение $\mathrm{R}$ из класса $R F$ имеет вид $\mathrm{R}_{1} \mathrm{c} * \mathrm{R}_{2}$, где $\mathrm{R}_{1}$ u $\mathrm{R}_{2}$ - выражения, задающ, языки $L\left(\mathrm{R}_{1}\right)=\left\{\alpha_{1}, \ldots, \alpha_{n_{1}}\right\}$ u $L\left(\mathrm{R}_{2}\right)=\left\{\beta_{1}, \ldots, \beta_{n_{2}}\right\}$, $\left|\alpha_{i}\right|=m_{1},\left|\beta_{j}\right|=m_{2}, a L(\mathrm{c})=\left\{a_{1}, \ldots, a_{k}\right\} \subset \Sigma, k<|\Sigma|$. Тогдa

$$
C_{l}\left(L^{p f}(. * \mathrm{R})\right)=\sum_{i=m}^{l}\left(\frac{k}{|\Sigma|}\right)^{i-m}-\varepsilon(l)
$$

где $|\varepsilon(l)| \leqslant \frac{n \cdot(l+1-2 m)}{|\Sigma|^{m-2} \cdot(|\Sigma|-k)^{2}}$ nри $l \geqslant 2 m$ и $\varepsilon(l)=0$ в остальных случалх, $m=m_{1}+m_{2}, n=n_{1} \cdot n_{2}$.

Доказательство. Очевидно, что $\min _{\alpha \in L(\mathrm{R})}=m_{1}+m_{2}$ и $G_{m_{1}+m_{2}}(L(\mathrm{R}))=$ $=G_{m_{1}}\left(L\left(\mathrm{R}_{1}\right)\right) \cdot G_{m_{2}}\left(L\left(\mathrm{R}_{2}\right)\right)=n_{1} \cdot n_{2} . \quad$ Тогда $C_{l}(L(\mathrm{R}))=$ $=\frac{G_{l}(L(\mathrm{R}))}{|\Sigma|^{l-m} \cdot n}=\left(\frac{k}{|\Sigma|}\right)^{l-m}$ при $l \geqslant m$ и $C_{l}(L(\mathrm{R}))=0$ при $l<m$.

Поэтому при $m \leqslant l<2 m$ верно равенство

$$
C_{l}\left(L^{p f}(. * \mathrm{R})\right)=C_{l-1}\left(L^{p f}(. * \mathrm{R})\right)+\left(\frac{k}{|\Sigma|}\right)^{l-m}
$$


При $l \geqslant 2 m$ верно следующее равенство:

$$
\begin{aligned}
& C_{l}\left(L^{p f}(. * \mathrm{R})\right)= \\
& =C_{l-1}\left(L^{p f}(* \mathrm{R})\right)+\left(\frac{k}{|\Sigma|}\right)^{l-m}-\frac{n}{|\Sigma|^{m}} \cdot \sum_{i=m}^{l-m}\left(\frac{k}{|\Sigma|}\right)^{i-m} \cdot C_{l-i}\left(L^{p f}(* \mathrm{R})\right)= \\
& =C_{l-1}\left(L^{p f}(* \mathrm{R})\right)+\left(\frac{k}{|\Sigma|}\right)^{l-m}-\frac{n}{|\Sigma|^{m}} \cdot \sum_{i=m+1}^{l-m}\left(\frac{k}{|\Sigma|}\right)^{i-m} \cdot C_{l-i}\left(L^{p f}(. * \mathrm{R})\right)- \\
& -\frac{n}{|\Sigma|^{m}} \cdot C_{l-m}\left(L^{p f}(. * \mathrm{R})\right)=C_{l-1}\left(L^{p f}(. * \mathrm{R})\right)+\left(\frac{k}{|\Sigma|}\right)^{l-m}- \\
& -\frac{k}{|\Sigma|} \cdot \frac{n}{|\Sigma|^{m}} \cdot \sum_{i=m}^{l-1-m}\left(\frac{k}{|\Sigma|}\right)^{i-m} \cdot C_{l-1-i}\left(L^{p f}(* \mathrm{R})\right)-\frac{n}{|\Sigma|^{m}} \cdot C_{l-m}\left(L^{p f}(. * \mathrm{R})\right)= \\
& =C_{l-1}\left(L^{p f}(. * \mathrm{R})\right)+\left(\frac{k}{|\Sigma|}\right)^{l-m}-\frac{k}{|\Sigma|} \frac{n}{|\Sigma|^{m}} \cdot \sum_{i=m}^{l-1-m}\left(\frac{k}{|\Sigma|}\right)^{i-m} \cdot C_{l-1-i}\left(L^{p f}(* \mathrm{R})\right)- \\
& -\frac{n}{|\Sigma|^{m}} \cdot\left(C_{l-1}\left(L^{p f}(. * \mathrm{R})\right)-C_{l-2}\left(L^{p f}(* \mathrm{R})\right)-\left(\frac{k}{|\Sigma|}\right)^{l-1-m}\right)= \\
& =C_{l-1}\left(L^{p f}(. * \mathrm{R})\right)+\frac{k}{|\Sigma|} \cdot\left(C_{l-1}\left(L^{p f}(. * \mathrm{R})\right)-C_{l-2}\left(L^{p f}(. * \mathrm{R})\right)\right)- \\
& -\frac{n}{|\Sigma|^{m}} \cdot C_{l-m}\left(L^{p f}(. * \mathrm{R})\right) .
\end{aligned}
$$

Обозначим через $\Delta(l)$ разность $C_{l}\left(L^{p f}(. * \mathrm{R})\right)-C_{l-1}\left(L^{p f}(. * \mathrm{R})\right)$. Тогда $\Delta(l)=\left(\frac{k}{|\Sigma|}\right)^{l-m}$ при $m \leqslant l<2 m$ и $\Delta(l)=\frac{k}{|\Sigma|} \cdot \Delta(l-1)-\frac{n}{|\Sigma|^{m}}$. $\cdot C_{l-m}\left(L^{p f}(. * \mathrm{R})\right)$ при $l \geqslant 2 m$. В силу неотрицательности $C_{l-m}\left(L^{p f}(. * \mathrm{R})\right)$ верно неравенство $\Delta(l) \leqslant\left(\frac{k}{|\Sigma|}\right)^{l-m}$. Тогда

$$
C_{l}\left(L^{p f}(. * \mathrm{R})\right)=\sum_{i=m}^{l} \Delta(i) \leqslant \sum_{i=m}^{l}\left(\frac{k}{|\Sigma|}\right)^{i-m}=\frac{1-\left(\frac{k}{|\Sigma|}\right)^{l+1-m}}{1-\frac{k}{|\Sigma|}}<\frac{|\Sigma|}{|\Sigma|-k} .
$$

Оценим $\Delta(l)$ снизу при $l \geqslant 2 m$ :

$$
\begin{aligned}
& \Delta(l) \geqslant\left(\frac{k}{|\Sigma|}\right) \cdot \Delta(l-1)-\frac{n}{|\Sigma|^{m}} \cdot \frac{|\Sigma|}{|\Sigma|-k} \geqslant \\
& \geqslant\left(\frac{k}{|\Sigma|}\right)^{l+1-2 m} \cdot \Delta(2 m-1)-\frac{n}{|\Sigma|^{m}} \cdot \frac{|\Sigma|}{|\Sigma|-k} \cdot \sum_{i=0}^{l-2 m}\left(\frac{k}{|\Sigma|}\right)^{i}= \\
& =\left(\frac{k}{|\Sigma|}\right)^{l-m}-\frac{n}{|\Sigma|^{m-1} \cdot(|\Sigma|-k)} \cdot \frac{1-\left(\frac{k}{|\Sigma|}\right)^{l+1-2 m}}{1-\frac{k}{|\Sigma|}} .
\end{aligned}
$$


Теперь можно оценить $C_{l}\left(L^{p f}(. * \mathrm{R})\right)$ снизу при $l \geqslant 2 m$ :

$$
\begin{aligned}
& C_{l}\left(L^{p f}(* \mathrm{R})\right)=C_{2 m-1}\left(L^{p f}(* \mathrm{R})\right)+\sum_{i=2 m}^{l} \Delta(i) \geqslant \sum_{i=m}^{2 m-1}\left(\frac{k}{|\Sigma|}\right)^{i-m}+ \\
& \quad+\sum_{i=2 m}^{l}\left(\frac{k}{|\Sigma|}\right)^{i-m}-\frac{n}{|\Sigma|^{m-1} \cdot(|\Sigma|-k)} \cdot \sum_{i=2 m}^{l} \frac{1-\left(\frac{k}{|\Sigma|}\right)^{i+1-2 m}}{1-\frac{k}{|\Sigma|}}= \\
& =\sum_{i=m}^{l}\left(\frac{k}{|\Sigma|}\right)^{i-m}-\frac{n}{|\Sigma|^{m-2} \cdot(|\Sigma|-k)^{2}} \cdot \sum_{i=2 m}^{l}\left(1-\left(\frac{k}{|\Sigma|}\right)^{i+1-2 m}\right)= \\
& =\sum_{i=m}^{l}\left(\frac{k}{|\Sigma|}\right)^{i-m}-\varepsilon^{\prime}(l),
\end{aligned}
$$

где $\left|\varepsilon^{\prime}(l)\right| \leqslant \frac{n \cdot(l+1-2 m)}{|\Sigma|^{m-2} \cdot(|\Sigma|-k)^{2}}$. Это неравенство можно обобщить на $m \leqslant l<2 m$, приняв на данном промежутке $\varepsilon^{\prime}(l)=0$.

Тогда при $l \geqslant m$ верно неравенство

$$
\sum_{i=m}^{l}\left(\frac{k}{|\Sigma|}\right)^{i-m}-\varepsilon^{\prime}(l) \leqslant C_{l}\left(L^{p f}(. * \mathrm{R})\right) \leqslant \sum_{i=m}^{l}\left(\frac{k}{|\Sigma|}\right)^{i-m}
$$

Лемма 5. Пусть регулярное выражение $\mathrm{R}$ из класса $R C \cap R F$ имеет вид $\mathrm{R}_{1} \mathrm{c}_{1} * \mathrm{R}_{2} \mathrm{c}_{2} * \ldots \mathrm{c}_{\mathrm{p}} * \mathrm{R}_{\mathrm{p}+1}$, где $\mathrm{R}_{\mathrm{i}}-$ выражения, задаюшие языки $L\left(\mathrm{R}_{\mathrm{i}}\right)=$ $=\left\{\alpha_{1}^{i}, \ldots, \alpha_{n_{i}}^{i}\right\},\left|\alpha_{j}^{i}\right|=m_{i}$ nри всех $j, a L\left(\mathrm{c}_{\mathrm{i}}\right)=\left\{a_{1}^{i}, \ldots, a_{k_{i}}^{i}\right\} \subset \Sigma, k_{i}<|\Sigma|$. Тогда верно следующее равенство:

$$
C_{l}\left(L^{p f}(. * \mathrm{R})\right)=(1-\varepsilon(l)) \cdot \sum_{i=m}^{l} C_{i}(L(\mathrm{R})),
$$

где $|\varepsilon(l)| \leqslant \frac{n \cdot(l+1-2 m)}{|\Sigma|^{m}} \cdot \prod_{i=1}^{p} \frac{|\Sigma|}{|\Sigma|-k_{i}}$ nри $l \geqslant 2 m u \varepsilon(l)=0$ в остальных случаях, $m=\sum_{i=1}^{p+1} m_{i}, n=\prod_{i=1}^{p+1} n_{i}$

Доказательство. Из равенства (2) следует верхняя оценка:

$$
C_{l}\left(L^{p f}(. * \mathrm{R})\right) \leqslant C_{l-1}\left(L^{p f}(. * \mathrm{R})\right)+C_{l}(L(\mathrm{R})) \leqslant \sum_{i=m}^{l} C_{i}(L(\mathrm{R})) .
$$


Оценим функцию роста $G_{l}(L(\mathrm{R}))$. Из условия следует равенство $L(\mathrm{R})=$ $=L\left(\mathrm{R}_{1}\right) L\left(\mathrm{c}_{1}\right)^{*} L\left(\mathrm{R}_{2}\right) L\left(\mathrm{c}_{2}\right)^{*} \ldots L\left(\mathrm{c}_{\mathrm{p}}\right)^{*} L\left(\mathrm{R}_{\mathrm{p}+1}\right)$. Тогда

$$
\begin{aligned}
& G_{l}(L(\mathrm{R}))= G_{l}\left(L\left(\mathrm{R}_{1}\right) L\left(\mathrm{c}_{1}\right)^{*} L\left(\mathrm{R}_{2}\right) L\left(\mathrm{c}_{2}\right)^{*} \ldots L\left(\mathrm{c}_{\mathrm{p}}\right)^{*} L\left(\mathrm{R}_{\mathrm{p}+1}\right)\right)= \\
&=G_{l}\left(\bigcup_{i_{1}+\ldots+i_{p}=l-m} L\left(\mathrm{R}_{1}\right) L\left(\mathrm{c}_{1}\right)^{i_{1}} L\left(\mathrm{R}_{2}\right) L\left(\mathrm{c}_{2}\right)^{i_{2}} \ldots L\left(\mathrm{c}_{\mathrm{p}}\right)^{i_{p}} L\left(\mathrm{R}_{\mathrm{p}+1}\right)\right) \leqslant \\
& \leqslant \sum_{i_{1}+\ldots+i_{p}=l-m}\left(\prod_{j=1}^{p+1} G_{m_{i}}\left(L\left(\mathrm{R}_{\mathrm{i}}\right)\right)\right) \cdot\left(\prod_{j=1}^{p} G_{i_{j}}\left(L\left(\mathrm{c}_{\mathrm{j}}\right)^{*}\right)\right) \leqslant \\
& \leqslant n \cdot \sum_{i_{1}+\ldots+i_{p}=l-m} k_{k_{1}}^{i_{1}} \cdot \ldots \cdot k_{p}^{i_{p}} .
\end{aligned}
$$

Поэтому верно неравенство $C_{l}(L(\mathrm{R})) \leqslant \frac{n \cdot \sum_{i_{1}+\ldots+i_{p}=l-m} k_{1}^{i_{1}} \cdot \ldots \cdot k_{p}{ }^{i_{p}}}{|\Sigma|^{l-m} \cdot n}=$ $=\sum_{i_{1}+\ldots+i_{p}=l-m}\left(\frac{k_{1}}{|\Sigma|}\right)^{i_{1}} \cdot \ldots \cdot\left(\frac{k_{p}}{|\Sigma|}\right)^{i_{p}}$. Тогда можно получить более слабую оценку для $C_{l}\left(L^{p f}(. * \mathrm{R})\right)$ :

$$
\begin{aligned}
C_{l}\left(L^{p f}(. * \mathrm{R})\right) \leqslant \sum_{i=m}^{l} C_{i}(L(\mathrm{R})) \leqslant \sum_{i=m}^{l} \sum_{i_{1}+\ldots+i_{p}=i-m}\left(\frac{k_{1}}{|\Sigma|}\right)^{i_{1}} \cdot \ldots \cdot\left(\frac{k_{p}}{|\Sigma|}\right)^{i_{p}}= \\
=\sum_{i=0}^{l-m} \sum_{i_{1}+\ldots+i_{p}=i}\left(\frac{k_{1}}{|\Sigma|}\right)^{i_{1}} \ldots \cdot\left(\frac{k_{p}}{|\Sigma|}\right)^{i_{p}} \leqslant \prod_{j=1}^{p}\left(\sum_{i=0}^{l-m}\left(\frac{k_{j}}{|\Sigma|}\right)^{i}\right)= \\
=\prod_{j=1}^{p}\left(\frac{1-\left(\frac{k_{j}}{|\Sigma|}\right)^{l+1-m}}{1-\frac{k_{j}}{|\Sigma|}}\right) \leqslant \prod_{j=1}^{p} \frac{|\Sigma|}{|\Sigma|-k_{j}} .
\end{aligned}
$$

Оценим $C_{l}\left(L^{p f}(. * \mathrm{R})\right)$ снизу при $l \geqslant 2 m$ :

$$
\begin{aligned}
C_{l}\left(L^{p f}(. * \mathrm{R})\right) \geqslant & C_{l-1}\left(L^{p f}(* \mathrm{R})\right)+C_{l}(L(\mathrm{R}))- \\
& -\frac{n}{|\Sigma|^{m}} \cdot \sum_{i=m}^{l-m} C_{i}(L(\mathrm{R})) \cdot \prod_{j=1}^{p} \frac{|\Sigma|}{|\Sigma|-k_{j}} \geqslant \sum_{i=m}^{l} C_{i}(L(\mathrm{R}))- \\
& -\frac{n}{|\Sigma|^{m}} \cdot \prod_{j=1}^{p} \frac{|\Sigma|}{|\Sigma|-k_{j}} \cdot \sum_{i=2 m}^{l} \sum_{j=m}^{i-m} C_{j}(L(\mathrm{R}))=\sum_{i=m}^{l} C_{i}(L(\mathrm{R}))- \\
& -\frac{n}{|\Sigma|^{m}} \cdot \prod_{j=1}^{p} \frac{|\Sigma|}{|\Sigma|-k_{j}} \cdot \sum_{i=m}^{l-m} C_{i}(L(\mathrm{R})) \cdot(l+1-m-i) \geqslant \\
& \geqslant \sum_{i=m}^{l} C_{i}(L(\mathrm{R})) \cdot\left(1-\frac{n \cdot(l+1-2 m)}{|\Sigma|^{m}} \cdot \prod_{j=1}^{p} \frac{|\Sigma|}{|\Sigma|-k_{j}}\right) .
\end{aligned}
$$


Таким образом верна оценка

$$
\left(1-\varepsilon^{\prime}(l)\right) \cdot \sum_{i=m}^{l} C_{i}(L(\mathrm{R})) \leqslant C_{l}\left(L^{p f}(. * \mathrm{R})\right) \leqslant \sum_{i=m}^{l} C_{i}(L(\mathrm{R}))
$$

где $\left|\varepsilon^{\prime}(l)\right|=\frac{n \cdot(l+1-2 m)}{|\Sigma|^{m}} \cdot \prod_{j=1}^{p} \frac{|\Sigma|}{|\Sigma|-k_{j}}$ при $l \geqslant 2 m$ и $\varepsilon^{\prime}(l)=0$ в остальных случаях.

Лемма 6. Пусть регулярное выражение $\mathrm{R}$ из класса $R C \cap R F$ имеет вид $\mathrm{R}_{1} \mathrm{c}_{1} * \mathrm{R}_{2} \mathrm{c}_{2} * \ldots \mathrm{c}_{\mathrm{p}} * \mathrm{R}_{\mathrm{p}+1}$, где $\mathrm{R}_{\mathrm{i}}$ - такие выражения, что регулярные языки $L\left(\mathrm{R}_{\mathrm{i}}\right)$ конечны, а $L\left(\mathrm{c}_{\mathrm{i}}\right)=\left\{a_{1}^{i}, \ldots, a_{k_{i}}^{i}\right\} \subset \Sigma, k_{i}<|\Sigma|$. Тогда

$$
C_{l}\left(L^{p f}(. * \mathrm{R})\right)=(1-\varepsilon(l)) \cdot \sum_{i=m}^{l} C_{i}(L(\mathrm{R})),
$$

где $|\varepsilon(l)| \leqslant \frac{G_{m}(L(\mathrm{R})) \cdot(l+1-2 m)}{|\Sigma|^{m}} \cdot C^{\max }\left(L^{p f}(. * \mathrm{R})\right)$ npu $l \geqslant 2 m u \varepsilon(l)=0$ в остальных случаях, $m_{i}=\min _{\alpha \in L\left(\mathrm{R}_{\mathrm{i}}\right)}|\alpha|, m=\min _{\alpha \in L(\mathrm{R})}|\alpha|=\sum_{i=1}^{p+1} m_{i}$.

Доказательство. Доказательство аналогично доказательству леммы 5.

Оценим функцию роста $G_{l}(L(\mathrm{R}))$ сверху:

$$
\begin{aligned}
& G_{l}(L(\mathrm{R}))=G_{l}\left(L\left(\mathrm{R}_{1}\right) L\left(\mathrm{c}_{1}\right)^{*} L\left(\mathrm{R}_{2}\right) L\left(\mathrm{c}_{2}\right)^{*} \ldots L\left(\mathrm{c}_{\mathrm{p}}\right)^{*} L\left(\mathrm{R}_{\mathrm{p}+1}\right)\right)= \\
& =G_{l}\left(\bigcup_{\substack{m \leqslant i \leqslant l \\
j_{1}+\ldots+j_{p+1}=i \\
i_{1}+\ldots+i_{p}=l-i}}\left(L\left(\mathrm{R}_{1}\right) \cap \Sigma^{j_{1}}\right) L\left(\mathrm{c}_{1}\right)^{i_{1}} \ldots L\left(\mathrm{c}_{\mathrm{p}}\right)^{i_{p}}\left(L\left(\mathrm{R}_{\mathrm{p}+1}\right) \cap \Sigma^{j_{p+1}}\right)\right) \leqslant \\
& \leqslant \sum_{i=m}^{l}\left(\sum_{\substack{i_{1}+\ldots+i_{p+1}=i \\
=}} \sum_{i=1}^{p+1} G_{i_{j}}\left(L\left(\mathrm{R}_{\mathrm{j}}\right)\right) \sum_{i_{1}+\ldots+i_{p}=l-i} \prod_{j=1}^{p} G_{i_{j}}\left(L\left(\mathrm{c}_{\mathrm{j}}\right)^{*}\right)=\right. \\
& \sum_{i_{1}+\ldots+i_{p+1}=i} \prod_{j=1}^{p+1} G_{i_{j}}\left(L\left(\mathrm{R}_{\mathrm{j}}\right)\right) \sum_{i_{1}+\ldots+i_{p}=l-i} k_{1}^{i_{1}} \cdot \ldots \cdot k_{p}^{i_{p}} .
\end{aligned}
$$




$$
\begin{aligned}
& \text { Обозначим } F(i)=\sum_{i_{1}+\ldots+i_{p+1}=i} \prod_{j=1}^{p+1} G_{i_{j}}\left(L\left(\mathrm{R}_{\mathrm{j}}\right)\right) \text { и оценим } C_{l}\left(L^{p f}(. * \mathrm{R})\right) \text { : } \\
& C_{l}\left(L^{p f}(* \mathrm{R})\right) \leqslant \sum_{i=m}^{l} \frac{G_{i}(L(\mathrm{R}))}{|\Sigma|^{i-m} \cdot G_{m}(L(\mathrm{R}))} \leqslant \\
& \leqslant \sum_{i=m}^{l} \sum_{j=m}^{i} \frac{F(j)}{|\Sigma|^{i-m} \cdot G_{m}(L(\mathrm{R}))} \sum_{i_{1}+\ldots+i_{p}=i-j} k_{1}^{i_{1}} \cdot \ldots \cdot k_{p}^{i_{p}}= \\
& =\sum_{i=m}^{l} \sum_{j=m}^{i} \frac{F(j)}{|\Sigma|^{j-m} \cdot G_{m}(L(\mathrm{R}))} \sum_{i_{1}+\ldots+i_{p}=i-j}\left(\frac{k_{1}}{|\Sigma|}\right)^{i_{1}} \ldots \cdot\left(\frac{k_{p}}{|\Sigma|}\right)^{i_{p}}= \\
& =\sum_{j=m}^{l} \frac{F(j)}{|\Sigma|^{j-m} \cdot G_{m}(L(\mathrm{R}))} \sum_{i=j}^{l} \sum_{i_{1}+\ldots+i_{p}=i-j}\left(\frac{k_{1}}{|\Sigma|}\right)^{i_{1}} \ldots \cdot\left(\frac{k_{p}}{|\Sigma|}\right)^{i_{p}}= \\
& =\sum_{j=m}^{l} \frac{\sum_{i_{1}+\ldots+i_{p+1}=j} \prod_{k=1}^{p+1} G_{i_{k}}\left(L\left(\mathrm{R}_{\mathrm{k}}\right)\right)}{|\Sigma|^{j} \cdot \prod_{k=1}^{p+1}|\Sigma|^{-m_{k}} \cdot G_{m_{k}}\left(L\left(\mathrm{R}_{\mathrm{k}}\right)\right)} \sum_{i=0}^{l-j} \sum_{i_{1}+\ldots+i_{p}=i}\left(\frac{k_{1}}{|\Sigma|}\right)^{i_{1}} \ldots \cdot\left(\frac{k_{p}}{|\Sigma|}\right)^{i_{p}} \leqslant \\
& \leqslant\left(\sum_{j=m}^{l} \sum_{i_{1}+\ldots+i_{p+1}=j} \prod_{k=1}^{p+1} \frac{G_{i_{k}}\left(L\left(\mathrm{R}_{\mathrm{k}}\right)\right)}{|\Sigma|^{i_{k}-m_{k}} \cdot G_{m_{k}}\left(L\left(\mathrm{R}_{\mathrm{k}}\right)\right)}\right) \cdot\left(\prod_{i=1}^{p} \frac{|\Sigma|}{|\Sigma|-k_{i}}\right)= \\
& =\left(\sum_{j=m}^{l} \sum_{i_{1}+\ldots+i_{p+1}=j} \prod_{k=1}^{p+1} C_{i_{k}}\left(L\left(\mathrm{R}_{\mathrm{k}}\right)\right)\right) \cdot\left(\prod_{i=1}^{p} \frac{|\Sigma|}{|\Sigma|-k_{i}}\right) \leqslant \\
& \leqslant\left(\prod_{i=1}^{p+1} \sum_{j=m_{i}}^{\infty} C_{j}\left(L\left(\mathrm{R}_{\mathrm{i}}\right)\right)\right) \cdot\left(\prod_{i=1}^{p} \frac{|\Sigma|}{|\Sigma|-k_{i}}\right) \stackrel{\text { def }}{=} C^{\prime} .
\end{aligned}
$$

Оценим $C_{l}\left(L^{p f}(. * \mathrm{R})\right)$ снизу при $l \geqslant 2 m$ :

$$
\begin{aligned}
C_{l}\left(L^{p f}(. * \mathrm{R})\right) \geqslant & C_{l-1}\left(L^{p f}(* \mathrm{R})\right)+C_{l}(L(\mathrm{R}))-\frac{n}{|\Sigma|^{m}} \cdot \sum_{i=m}^{l-m} C_{i}(L(\mathrm{R})) \cdot C^{\prime} \geqslant \\
& \geqslant \sum_{i=m}^{l} C_{i}(L(\mathrm{R}))-\frac{n}{|\Sigma|^{m}} \cdot C^{\prime} \cdot \sum_{i=2 m}^{l} \sum_{j=m}^{i-m} C_{j}(L(\mathrm{R}))= \\
= & \sum_{i=m}^{l} C_{i}(L(\mathrm{R}))-\frac{n}{|\Sigma|^{m}} \cdot C^{\prime} \cdot \sum_{i=m}^{l-m} C_{i}(L(\mathrm{R})) \cdot(l+1-m-i) \geqslant \\
& \geqslant \sum_{i=m}^{l} C_{i}(L(\mathrm{R})) \cdot\left(1-\frac{n \cdot(l+1-2 m)}{|\Sigma|^{m}} \cdot C^{\prime}\right) .
\end{aligned}
$$

Таким образом,

$$
\left(1-\varepsilon^{\prime}(l)\right) \cdot \sum_{i=m}^{l} C_{i}(L(\mathrm{R})) \leqslant C_{l}\left(L^{p f}(. * \mathrm{R})\right) \leqslant \sum_{i=m}^{l} C_{i}(L(\mathrm{R})),
$$


где $\left|\varepsilon^{\prime}(l)\right|=\frac{G_{m}(L(\mathrm{R})) \cdot(l+1-2 m)}{|\Sigma|^{m}} \cdot\left(\prod_{i=1}^{p+1} \sum_{j=m_{i}}^{\infty} C_{j}\left(L\left(\mathrm{R}_{\mathrm{i}}\right)\right)\right) \cdot\left(\prod_{i=1}^{p} \frac{|\Sigma|}{|\Sigma|-k_{i}}\right)$ при $l \geqslant 2 m$ и $\varepsilon^{\prime}(l)=0$ в остальных случаях.

Лемма 7. Пусть $\mathrm{R}_{1}$ u $\mathrm{R}_{2}$ - регулярные выражения. Тогда

$$
L^{p f}\left(* \mathrm{R}_{1} * \mathrm{R}_{2}\right)=L^{p f}\left(* \mathrm{R}_{1}\right) L^{p f}\left(* \mathrm{R}_{2}\right) .
$$

Доказательство. Пусть $\alpha \in L^{p f}\left(. * \mathrm{R}_{1}\right) L^{p f}\left(. * \mathrm{R}_{2}\right)$, тогда слово $\alpha$ представимо в виде $\alpha=\alpha_{1} \alpha_{2}$, где $\alpha_{1} \in L^{p f}\left(. * \mathrm{R}_{1}\right) \subset L\left(. * \mathrm{R}_{1}\right), \quad \alpha_{2} \in L^{p f}\left(. * \mathrm{R}_{2}\right) \subset$ $\subset L\left(. * \mathrm{R}_{2}\right)$. Докажем от противного, что $\alpha \in L^{p f}\left(. * \mathrm{R}_{1} \cdot * \mathrm{R}_{2}\right)$. Заметим, что слово $\alpha \in L\left(. * \mathrm{R}_{1}\right) L\left(. * \mathrm{R}_{2}\right)=L\left(. * \mathrm{R}_{1} * * \mathrm{R}_{2}\right)$. Следовательно, если $L^{p f}\left(. * \mathrm{R}_{1} \cdot * \mathrm{R}_{2}\right)$ не содержит слов, отличных от всего слова $\alpha$, но являющихся префиксом $\alpha$, то слово $\alpha$ принадлежит языку $L^{p f}\left(. * \mathrm{R}_{1} * \mathrm{R}_{2}\right)$. Пусть найдется такое $\beta \in L^{p f}\left(. * \mathrm{R}_{1}, * \mathrm{R}_{2}\right)$, что $\alpha \neq \beta$ и слово $\beta$ является префиксом слова $\alpha$. Тогда, очевидно, слово $\beta$ представимо в виде $\beta=\beta_{1} \beta_{2}$, где $\beta_{1} \in L^{p f}\left(. * \mathrm{R}_{1}\right), \beta_{2} \in L\left(. * \mathrm{R}_{2}\right)$. По предположению слово $\beta$ является префиксом $\alpha$, следовательно, если $\left|\alpha_{1}\right| \neq\left|\beta_{1}\right|$, то либо слово $\alpha_{1} \in L^{p f}\left(. * \mathrm{R}_{1}\right)$ является префиксом слова $\beta_{1} \in L^{p f}\left(. * \mathrm{R}_{1}\right)$, либо слово $\beta_{1}$ является префиксом слова $\alpha_{1}$, что невозможно в силу определения $L^{p f}()$. Поэтому $\alpha_{1}=\beta_{1}$. Слово $\beta$ является отличным от $\alpha$ префиксом слова $\alpha$, а значит, в силу равенства $\alpha_{1}=\beta_{1}$, слово $\beta_{2} \in L\left(. * \mathrm{R}_{2}\right)$ является префиксом слова $\alpha_{2}$, причем $\alpha_{2} \neq \beta_{2}$. Но это противоречит тому, что $\alpha_{2} \in L^{p f}\left(. * \mathrm{R}_{2}\right)$. Следовательно, $L^{p f}\left(. * \mathrm{R}_{1}\right) L^{p f}\left(. * \mathrm{R}_{2}\right) \subseteq$ $\subseteq L^{p f}\left(. * \mathrm{R}_{1} * * \mathrm{R}_{2}\right)$.

Пусть $\alpha \in L^{p f}\left(. * \mathrm{R}_{1} * \mathrm{R}_{2}\right) \subset L\left(. * \mathrm{R}_{1} * \mathrm{R}_{2}\right)$. Тогда слово $\alpha$ представимо в виде $\alpha=\alpha_{1} \alpha 2$, где $\alpha_{1} \in L^{p f}\left(. * \mathrm{R}_{1}\right)$, а $\alpha_{2} \in L\left(. * \mathrm{R}_{2}\right)$. Докажем от противного, что $\alpha_{2} \in L^{p f}\left(. * \mathrm{R}_{2}\right)$. Пусть $\alpha_{2} \notin L^{p f}\left(. * \mathrm{R}_{2}\right)$; тогда найдется слово $\alpha_{2} \in L^{p f}\left(. * \mathrm{R}_{2}\right)$, являющееся префиксом слова $\alpha_{2}$. Обозначим через $\alpha^{\prime}$ конкатенацию слов $\alpha_{1} \alpha_{2}^{\prime}$. По построению и по доказанному выше $\alpha^{\prime} \in L^{p f}\left(. * \mathrm{R}_{1}\right) L^{p f}\left(. * \mathrm{R}_{2}\right) \subseteq L^{p f}\left(. * \mathrm{R}_{1} * \mathrm{R}_{2}\right)$. Но тогда различные слова $\alpha$ и $\alpha_{1}$ принадлежат языку $L^{p f}\left(. * \mathrm{R}_{1}, * \mathrm{R}_{2}\right)$ и при этом слово $\alpha^{\prime}$ является префиксом слова $\alpha$. Противоречие определению $L^{p f}()$. Следовательно, $L^{p f}\left(. * \mathrm{R}_{1}, * \mathrm{R}_{2}\right)=L^{p f}\left(* \mathrm{R}_{1}\right) L^{p f}\left(. * \mathrm{R}_{2}\right)$.

Следствие 1. Пусть $\mathrm{R}_{1} u \mathrm{R}_{2}$ - регулярные выражения. Тогда верно равенство

$$
G_{l}\left(L^{p f}\left(. * \mathrm{R}_{1} \cdot * \mathrm{R}_{2}\right)\right)=\sum_{i=m_{1}}^{l-m_{2}} G_{i}\left(L^{p f}\left(* \mathrm{R}_{1}\right)\right) \cdot G_{l-i}\left(L^{p f}\left(* \mathrm{R}_{2}\right)\right),
$$

əде $m_{1}=\min _{\alpha \in L\left(\mathrm{R}_{1}\right)}|\alpha|, m_{2}=\min _{\alpha \in L\left(\mathrm{R}_{2}\right)}|\alpha|$.

Доказательство. По лемме 7 верно равенство $L^{p f}\left(* \mathrm{R}_{1} * \mathrm{R}_{2}\right)=$ $=L^{p f}\left(* \mathrm{R}_{1}\right) L^{p f}\left(* \mathrm{R}_{2}\right)$. Тогда

$$
\begin{aligned}
L^{p f}\left(. * \mathrm{R}_{1} * * \mathrm{R}_{2}\right) \cap \Sigma^{l}=\left(L^{p f}\left(. * \mathrm{R}_{1}\right) L^{p f}\right. & \left.\left(* \mathrm{R}_{2}\right)\right) \cap \Sigma^{l}= \\
& =\bigcup_{i=m_{1}}^{l-m_{2}}\left(L^{p f}\left(* \mathrm{R}_{1}\right) \cap \Sigma^{i}\right)\left(L^{p f}\left(. * \mathrm{R}_{2}\right) \cap \Sigma^{l-i}\right) .
\end{aligned}
$$

Докажем от противного, что множества $\left(L^{p f}\left(. * \mathrm{R}_{1}\right) \cap \Sigma^{i}\right)\left(L^{p f}\left(. * \mathrm{R}_{2}\right) \cap \Sigma^{l-i}\right)$ и $\left(L^{p f}\left(. * \mathrm{R}_{1}\right) \cap \Sigma^{j}\right)\left(L^{p f}\left(. * \mathrm{R}_{2}\right) \cap \Sigma^{l-j}\right)$ не пересекаются при $i \neq j$. Действительно, пусть $\alpha$ лежит в пересечении $\left(L^{p f}\left(. * \mathrm{R}_{1}\right) \cap \Sigma^{i}\right)\left(L^{p f}\left(. * \mathrm{R}_{2}\right) \cap \Sigma^{l-i}\right) \cap$ 
$\cap\left(L^{p f}\left(* \mathrm{R}_{1}\right) \cap \Sigma^{j}\right)\left(L^{p f}\left(* \mathrm{R}_{2}\right) \cap \Sigma^{l-j}\right)$. Тогда слово $\alpha$ представимо в виде $\alpha=$ $=\alpha_{1} \alpha_{2}=\alpha_{1}^{\prime} \alpha_{2}^{\prime}, \quad$ где $\alpha_{1} \in\left(L^{p f}\left(. * \mathrm{R}_{1}\right) \cap \Sigma^{i}\right), \quad \alpha_{1}^{\prime} \in\left(L^{p f}\left(* \mathrm{R}_{1}\right) \cap \Sigma^{j}\right), \quad \alpha_{2} \in$ $\in\left(L^{p f}\left(. * \mathrm{R}_{2}\right) \cap \Sigma^{l-i}\right)$ и $\alpha_{2}^{\prime} \in\left(L^{p f}\left(* \mathrm{R}_{2}\right) \cap \Sigma^{l-j}\right)$. Однако в силу того, что $i \neq j$, либо слово $\alpha_{1} \in L^{p f}\left(. * \mathrm{R}_{1}\right)$ является нетривиальным префиксом слова $\alpha_{1}^{\prime} \in L^{p f}\left(* \mathrm{R}_{1}\right)$, либо $\alpha_{1}^{\prime}$ является нетривиальным префиксом слова $\alpha_{1}$. Противоречие. Следовательно, $\left(L^{p f}\left(. * \mathrm{R}_{1}\right) \cap \Sigma^{i}\right)\left(L^{p f}\left(* \mathrm{R}_{2}\right) \cap \Sigma^{l-i}\right) \cap$ $\cap\left(L^{p f}\left(. * \mathrm{R}_{1}\right) \cap \Sigma^{j}\right)\left(L^{p f}\left(* \mathrm{R}_{2}\right) \cap \Sigma^{l-j}\right)=\varnothing$ при $i \neq j$.

Из доказанного выше следует равенство:

$$
\begin{aligned}
G_{l}\left(L^{p f}\left(. * \mathrm{R}_{1} * \mathrm{R}_{2}\right)\right) & =\left|L^{p f}\left(* \mathrm{R}_{1} * * \mathrm{R}_{2}\right) \cap \Sigma^{l}\right|= \\
& =\left|\bigcup_{i=m_{1}}^{l-m_{2}}\left(L^{p f}\left(* \mathrm{R}_{1}\right) \cap \Sigma^{i}\right)\left(L^{p f}\left(* \mathrm{R}_{2}\right) \cap \Sigma^{l-i}\right)\right|= \\
& =\sum_{i=m_{1}}^{l-m_{2}}\left|\left(L^{p f}\left(* \mathrm{R}_{1}\right) \cap \Sigma^{i}\right)\left(L^{p f}\left(* \mathrm{R}_{2}\right) \cap \Sigma^{l-i}\right)\right|= \\
& =\sum_{i=m_{1}}^{l-m_{2}} G_{i}\left(L^{p f}\left(* \mathrm{R}_{1}\right)\right) \cdot G_{l-i}\left(L^{p f}\left(* \mathrm{R}_{2}\right)\right) .
\end{aligned}
$$

Лемма 8. Пусть $\mathrm{R}_{1}$ u $\mathrm{R}_{2}$ - регулярные выражения. Тогда

$$
\begin{aligned}
G_{l}\left(L^{p f}\left(* \mathrm{R}_{1} * \mathrm{R}_{2} *\right)\right)=|\Sigma|^{l-m_{1}-m_{2}} \cdot G_{m_{1}}\left(L\left(\mathrm{R}_{1}\right)\right) \cdot G_{m_{2}}\left(L\left(\mathrm{R}_{2}\right)\right) \cdot & \\
& \cdot \sum_{i+j \leqslant l} C_{i}\left(L^{p f}\left(. * \mathrm{R}_{1}\right)\right) \cdot C_{j}\left(L^{p f}\left(. * \mathrm{R}_{2}\right)\right),
\end{aligned}
$$

где $m_{1}=\min _{\alpha \in L\left(\mathrm{R}_{1}\right)}|\alpha|, m_{2}=\min _{\alpha \in L\left(\mathrm{R}_{2}\right)}|\alpha|$.

Доказательство. По лемме 1

$$
G_{l}\left(L\left(. * \mathrm{R}_{1} * \mathrm{R}_{2} * *\right)\right)=\sum_{i=m_{1}+m_{2}}^{l} G_{i}\left(L^{p f}\left(* \mathrm{R}_{1} * \mathrm{R}_{2}\right)\right) \cdot|\Sigma|^{l-i}
$$

Тогда по следствию из леммы 7

$$
\begin{gathered}
G_{l}\left(L\left(* \mathrm{R}_{1} * \mathrm{R}_{2} *\right)\right)=\sum_{i=m_{1}+m_{2}}^{l} \sum_{j=m_{1}}^{i-m_{2}} G_{j}\left(L^{p f}\left(* \mathrm{R}_{1}\right)\right) \cdot G_{i-j}\left(L^{p f}\left(* \mathrm{R}_{2}\right)\right) \cdot|\Sigma|^{l-i}= \\
=\sum_{i=m_{1}+m_{2}}^{l} \sum_{j=m_{1}}^{i-m_{2}}|\Sigma|^{j-m_{1}} \cdot G_{m_{1}}\left(L\left(\mathrm{R}_{1}\right)\right) \cdot C_{j}\left(L^{p f}\left(* \mathrm{R}_{1}\right)\right) \cdot|\Sigma|^{i-j-m_{2}} . \\
\cdot G_{m_{2}}\left(L\left(\mathrm{R}_{2}\right)\right) \cdot C_{i-j}\left(L^{p f}\left(* \mathrm{R}_{2}\right)\right) \cdot|\Sigma|^{l-i}=|\Sigma|^{l-m_{1}-m_{2}} \cdot G_{m_{1}}\left(L\left(\mathrm{R}_{1}\right)\right) \cdot \\
\cdot G_{m_{2}}\left(L\left(\mathrm{R}_{2}\right)\right) \cdot \sum_{i=m_{1}+m_{2}}^{l} \sum_{j=m_{1}}^{i-m_{2}} C_{j}\left(L^{p f}\left(* \mathrm{R}_{1}\right)\right) \cdot C_{i-j}\left(L^{p f}\left(* \mathrm{R}_{2}\right)\right)= \\
=|\Sigma|^{l-m_{1}-m_{2}} \cdot G_{m_{1}}\left(L\left(\mathrm{R}_{1}\right)\right) \cdot G_{m_{2}}\left(L\left(\mathrm{R}_{2}\right)\right) \cdot \\
\cdot \sum_{i+j \leqslant l} C_{i}\left(L^{p f}\left(* \mathrm{R}_{1}\right)\right) \cdot C_{j}\left(L^{p f}\left(* \mathrm{R}_{2}\right)\right) .
\end{gathered}
$$




\section{6. Доказательство теоремы 1}

Обозначим через $\mathrm{R}^{\mathrm{ij}}$ регулярное выражение $* \mathrm{R}_{\mathrm{i}} * \mathrm{R}_{\mathrm{j}} *$. В силу того, что

$$
\begin{aligned}
& L\left(. *\left(\mathrm{R}_{1} \mid \mathrm{R}_{3}\right) \cdot *\left(\mathrm{R}_{2} \mid \mathrm{R}_{4}\right) \cdot *\right)= \\
& \qquad \begin{aligned}
L\left(* \mathrm{R}_{1} \cdot * \mathrm{R}_{2} \cdot *\right) \cup L\left(* \mathrm{R}_{3} \cdot * \mathrm{R}_{4} * *\right) \cup L\left(* \mathrm{R}_{1} * \mathrm{R}_{4} \cdot *\right) \cup \\
\qquad L\left(. * \mathrm{R}_{3} \cdot * \mathrm{R}_{2} \cdot *\right)=L\left(\mathrm{R}^{12}\right) \cup L\left(\mathrm{R}^{34}\right) \cup L\left(\mathrm{R}^{14}\right) \cup L\left(\mathrm{R}^{32}\right),
\end{aligned}
\end{aligned}
$$

верно следующее неравенство:

$$
\begin{aligned}
& \frac{G_{l}\left(L\left(* *\left(\mathrm{R}_{1} \mid \mathrm{R}_{3}\right) \cdot *\left(\mathrm{R}_{2} \mid \mathrm{R}_{4}\right) \cdot *\right)\right)}{G_{l}\left(L\left(\mathrm{R}^{12}\right) \cup L\left(\mathrm{R}^{34}\right)\right)} \leqslant \\
& \leqslant \frac{G_{l}\left(L\left(\mathrm{R}^{12}\right) \cup L\left(\mathrm{R}^{34}\right)\right)+G_{l}\left(L\left(\mathrm{R}^{14}\right)\right)+G_{l}\left(L\left(\mathrm{R}^{32}\right)\right)}{G_{l}\left(L\left(\mathrm{R}^{12}\right) \cup L\left(\mathrm{R}^{34}\right)\right)}= \\
& \quad=1+\frac{G_{l}\left(R^{14}\right)+G_{l}\left(L\left(\mathrm{R}^{32}\right)\right)}{G_{l}\left(L\left(\mathrm{R}^{12}\right) \cup L\left(\mathrm{R}^{34}\right)\right)} \leqslant 1+\frac{G_{l}\left(R^{14}\right)}{G_{l}\left(R^{34}\right)}+\frac{G_{l}\left(R^{32}\right)}{G_{l}\left(R^{34}\right)} .
\end{aligned}
$$

Оценим отношение $\frac{G_{l}\left(R^{14}\right)}{G_{l}\left(R^{34}\right)}$ при $l \geqslant 2 m_{3}$, используя леммы 5 и 8 :

$$
\begin{aligned}
& \frac{G_{l}\left(R^{14}\right)}{G_{l}\left(R^{34}\right)}=\frac{|\Sigma|^{l-m_{1}-m_{4}} \cdot G_{m_{1}}\left(L\left(\mathrm{R}_{1}\right)\right) \cdot G_{m_{4}}\left(L\left(\mathrm{R}_{4}\right)\right)}{|\Sigma|^{l-m_{3}-m_{4}} \cdot G_{m_{3}}\left(L\left(\mathrm{R}_{3}\right)\right) \cdot G_{m_{4}}\left(L\left(\mathrm{R}_{4}\right)\right)} . \\
& \frac{\sum_{i+j \leqslant l} C_{i}\left(L^{p f}\left(. * \mathrm{R}_{1}\right)\right) \cdot C_{j}\left(L^{p f}\left(. * \mathrm{R}_{4}\right)\right)}{\sum_{i+j \leqslant l} C_{i}\left(L^{p f}\left(. * \mathrm{R}_{3}\right)\right) \cdot C_{j}\left(L^{p f}\left(* \mathrm{R}_{4}\right)\right)}= \\
& =|\Sigma|^{m_{3}-m_{1}} \cdot \frac{G_{m_{1}}\left(L\left(\mathrm{R}_{1}\right)\right)}{G_{m_{3}}\left(L\left(\mathrm{R}_{3}\right)\right)} \cdot \frac{\sum_{i=m_{4}}^{l-m_{1}} C_{i}\left(L^{p f}\left(* \mathrm{R}_{4}\right)\right) \sum_{j=m_{1}}^{l-i} C_{j}\left(L^{p f}\left(* \mathrm{R}_{1}\right)\right)}{\sum_{i=m_{4}}^{l-m_{3}} C_{i}\left(L^{p f}\left(* \mathrm{R}_{4}\right)\right) \sum_{j=m_{3}}^{l-i} C_{j}\left(L^{p f}\left(* \mathrm{R}_{3}\right)\right)} \leqslant \\
& \leqslant|\Sigma|^{m_{3}-m_{1}} \cdot \frac{G_{m_{1}}\left(L\left(\mathrm{R}_{1}\right)\right)}{G_{m_{3}}\left(L\left(\mathrm{R}_{3}\right)\right)} \cdot \frac{\sum_{i=m_{4}}^{l-m_{3}} C_{i}\left(L^{p f}\left(* \mathrm{R}_{4}\right)\right) \sum_{j=m_{3}}^{l-i} C_{j}\left(L^{p f}\left(* \mathrm{R}_{1}\right)\right)}{\sum_{i=m_{4}}^{l-m_{3}} C_{i}\left(L^{p f}\left(* \mathrm{R}_{4}\right)\right) \sum_{j=m_{3}}^{l-i} C_{j}\left(L^{p f}\left(* \mathrm{R}_{3}\right)\right)} \leqslant \\
& \leqslant|\Sigma|^{m_{3}-m_{1}} \cdot \frac{G_{m_{1}}\left(L\left(\mathrm{R}_{1}\right)\right)}{G_{m_{3}}\left(L\left(\mathrm{R}_{3}\right)\right)} . \\
& \sum_{i=m_{4}}^{l-m_{3}} C_{i}\left(L^{p f}\left(. * \mathrm{R}_{4}\right)\right) \sum_{j=m_{3}}^{l-i} C^{\max }\left(L^{p f}\left(. * \mathrm{R}_{1}\right)\right) \\
& \overline{\sum_{i=m_{4}}^{l-m_{3}} C_{i}\left(L^{p f}\left(* \mathrm{R}_{4}\right)\right) \sum_{j=m_{3}}^{l-i}\left(1-\frac{G_{m_{3}}\left(L\left(\mathrm{R}_{3}\right)\right) \cdot\left(l+1-2 m_{3}\right)}{|\Sigma|^{m_{3}}} \cdot C^{\max }\left(L^{p f}\left(* \mathrm{R}_{3}\right)\right)\right)}= \\
& =|\Sigma|^{m_{3}-m_{1}} \cdot \frac{G_{m_{1}}\left(L\left(\mathrm{R}_{1}\right)\right)}{G_{m_{3}}\left(L\left(\mathrm{R}_{3}\right)\right)} \cdot \frac{C^{\max }\left(L^{p f}\left(. * \mathrm{R}_{1}\right)\right)}{1-\frac{G_{m_{3}}\left(L\left(\mathrm{R}_{3}\right)\right) \cdot\left(l+1-2 m_{3}\right)}{|\Sigma|^{m_{3}}} \cdot C^{\max }\left(L^{p f}\left(* \mathrm{R}_{3}\right)\right)} .
\end{aligned}
$$

Тогда при

$$
2 m_{3} \leqslant l \leqslant \frac{\varepsilon}{\frac{G_{m_{3}}\left(L\left(\mathrm{R}_{3}\right)\right)}{|\Sigma|^{m_{3}}} \cdot C^{\max }\left(L^{p f}\left(* \mathrm{R}_{3}\right)\right)}-1+2 m_{3}
$$


выполняется неравенство

$$
\frac{G_{l}\left(R^{14}\right)}{G_{l}\left(R^{34}\right)} \leqslant \frac{1}{1-\varepsilon} \cdot|\Sigma|^{m_{3}-m_{1}} \cdot \frac{G_{m_{1}}\left(L\left(\mathrm{R}_{1}\right)\right)}{G_{m_{3}}\left(L\left(\mathrm{R}_{3}\right)\right)} \cdot C^{\max }\left(L^{p f}\left(. * \mathrm{R}_{1}\right)\right) .
$$

Причем при $m_{3}+m_{4} \leqslant l<2 m_{3}$ неравенство (4) также, очевидно, верно. Заметим, что из леммы 8 следует равенство

$$
\frac{G_{l}\left(L\left(* \mathrm{R}_{3} * \mathrm{R}_{2} *\right)\right)}{G_{l}\left(L\left(. * \mathrm{R}_{3} * \mathrm{R}_{4} * *\right)\right)}=\frac{G_{l}\left(L\left(* \mathrm{R}_{2} * \mathrm{R}_{3} *\right)\right)}{G_{l}\left(L\left(* \mathrm{R}_{4} * \mathrm{R}_{3} *\right)\right)} .
$$

Поэтому при

$$
m_{3}+m_{4} \leqslant l \leqslant \frac{\varepsilon}{\frac{G_{m_{3}}\left(L\left(\mathrm{R}_{3}\right)\right)}{|\Sigma|^{m_{3}}} \cdot C^{\max }\left(L^{p f}\left(\mathrm{R}_{3}\right)\right)}-1+2 m_{3}
$$

аналогично неравенству (4) верно неравенство

$$
\frac{G_{l}\left(R^{32}\right)}{G_{l}\left(R^{34}\right)} \leqslant \frac{1}{1-\varepsilon} \cdot|\Sigma|^{m_{4}-m_{2}} \cdot \frac{G_{m_{2}}\left(L^{p f}\left(. * \mathrm{R}_{2}\right)\right)}{G_{m_{4}}\left(L^{p f}\left(. * \mathrm{R}_{4}\right)\right)} \cdot C^{\max }\left(L^{p f}\left(. * \mathrm{R}_{2}\right)\right) .
$$

Таким образом, подставив (4) и (5) в неравенство (3), получаем требуемое неравенство.

\section{7. Применение алгоритма}

Пусть задано $n$ регулярных выражений $\mathrm{R}^{\mathrm{i}}=. * \mathrm{R}_{2 \mathrm{i}-1} \cdot * \mathrm{R}_{2 \mathrm{i}} \cdot *$, где выражения $\mathrm{R}_{\mathrm{j}}$ $(j=\overline{1,2 n})$ принадлежат классу $R C \cap R F$. Для того, чтобы оценить относительный прирост числа слов длины не более $M$ при “слиянии" для каждой пары выражений $R^{i}$, достаточно вычислить значения выражений $m_{j}=$ $\min _{\alpha \in L\left(\mathrm{R}_{\mathrm{j}}\right)}|\alpha|, N_{j}=\frac{G_{m_{j}}\left(L\left(\mathrm{R}_{\mathrm{j}}\right)\right)}{|\Sigma|^{m_{j}}}, C_{j}^{\max }=C^{\max }\left(L^{p f}\left(* \mathrm{R}_{\mathrm{j}}\right)\right)$ при $j=\overline{1,2 n}$ и $\varepsilon_{i}=$ $M \cdot \max \left(C_{2 i-1}^{\max } \cdot N_{2 i-1}, C_{2 i}^{\max } \cdot N_{2 i}\right)$ при $i=\overline{1, n}$. Тогда для любой пары таких выражений $\mathrm{R}^{\mathrm{i}}$ и $\mathrm{R}^{\mathrm{j}}$ что $m_{2 i-1} \geqslant m_{2 j-1}, m_{2 i} \geqslant m_{2 j}$ и $\varepsilon_{j}<1$, относительный прирост слов длины не более $M$ при "слиянии" выражений можно оценить сверху величиной

$$
\frac{1}{1-\varepsilon_{j}} \cdot\left(\frac{C_{2 i-1}^{\max } \cdot N_{2 i-1}}{N_{2 j-1}}+\frac{C_{2 i}^{\max } \cdot N_{2 i}}{N_{2 j}}\right) .
$$

Отдельно отметим, что в случае $m_{2 i-1}>m_{2 j-1}$ и $m_{2 i}<m_{2 j}$ в исходном регулярном языке $L\left(\mathrm{R}^{\mathrm{i}}\right) \cup L\left(\mathrm{R}^{\mathrm{j}}\right)$ отсутствуют слова длины $m_{2 j-1}+m_{2 i}$, а в языке $L\left(. *\left(\mathrm{R}_{2 \mathrm{i}-1} \mid \mathrm{R}_{2 j-1}\right) . *\left(\mathrm{R}_{2 \mathrm{i}} \mid \mathrm{R}_{2 \mathrm{j}}\right) . *\right)$ они присутствуют в количестве $G_{m_{2 j-1}}\left(L\left(\mathrm{R}_{2 j-1}\right)\right)$. $G_{m_{2 i}}\left(L\left(\mathrm{R}_{2 \mathrm{i}}\right)\right)$.

Для исследования эффективности применения данного метода оценки были рассмотрены 36 регулярных выражений вида $\mathrm{R}^{\mathrm{i}}=. * \mathrm{R}_{2 \mathrm{i}-1} \cdot * \mathrm{R}_{2 \mathrm{i}} * *$ из базы сигнатур системы Snort [16]. Вычисление необходимых параметров, а также построение ДКА для пар выражений и модифицированных выражений осуществлялось с помощью разработанного для этих целей программного комплекса (свидетельство о государственной регистрации программы для ЭВМ №2014614857). Для каждого из 72 подвыражений $\mathrm{R}_{\mathrm{j}}$ были вычислены $m_{j}=\min _{\alpha \in L\left(\mathrm{R}_{\mathrm{j}}\right)}|\alpha|, N_{j}=\frac{G_{m_{j}}\left(L\left(\mathrm{R}_{\mathrm{j}}\right)\right)}{|\Sigma|^{m_{j}}}$ и $C_{j}^{\text {max }}=$ 
$C^{\max }\left(L^{p f}\left(* \mathrm{R}_{\mathrm{j}}\right)\right)$ при $j=\overline{1,2 n}$, а также для каждого из 36 выражений $\mathrm{R}^{\mathrm{i}}$ были вычислены значения $S_{i}=\max \left(C_{2 i-1}^{\max } \cdot N_{2 i-1}, C_{2 i}^{\max } \cdot N_{2 i}\right)$. В силу того, что для оценок необходимо, чтобы $\varepsilon_{i}$ не превосходили 1 , максимальная длина $M=\frac{\varepsilon_{i}}{S_{i}}$, для которой оценки применимы, не превосходит $\frac{1}{S_{i}}$. В трех случаях из 36 отношение $\frac{1}{S_{i}}$ имеет порядок $10^{3}$, а значит, оценка расширения регулярного языка при "слиянии" возможна только для относительно коротких слов длины не более 1000. В остальных 33 случаях отношение $\frac{1}{S_{i}}$ превосходит $2.8 \cdot 10^{8}$. Таким образом, при оценке, например, прироста в случае слов длины до $M=10^{6}$ (1MB данных) значения выражений $\varepsilon_{i}=M \cdot S_{i}$ не превосходят 0.00357. Среди 33 таких выражений 19 выражений принадлежат классу $R F$. Среди $C_{19}^{2}=171$ пар этих 19 выражений в 79 случаях минимальные длины $m_{i}$ таковы, что оценка (6) применима. Причем в двух случаях верхняя оценка относительного прироста числа слов не превышает 1, а в 66 случаях не превышает $10^{-2}$.

Кроме того, сравнение результатов применения описанного метода оценки к 33 выражениям системы Snort, у которых значение $\frac{1}{S_{i}}$ превосходит $2.8 \cdot 10^{8}$, и точных значений относительного роста числа слов длины $2^{20}$ показало, что предложенный

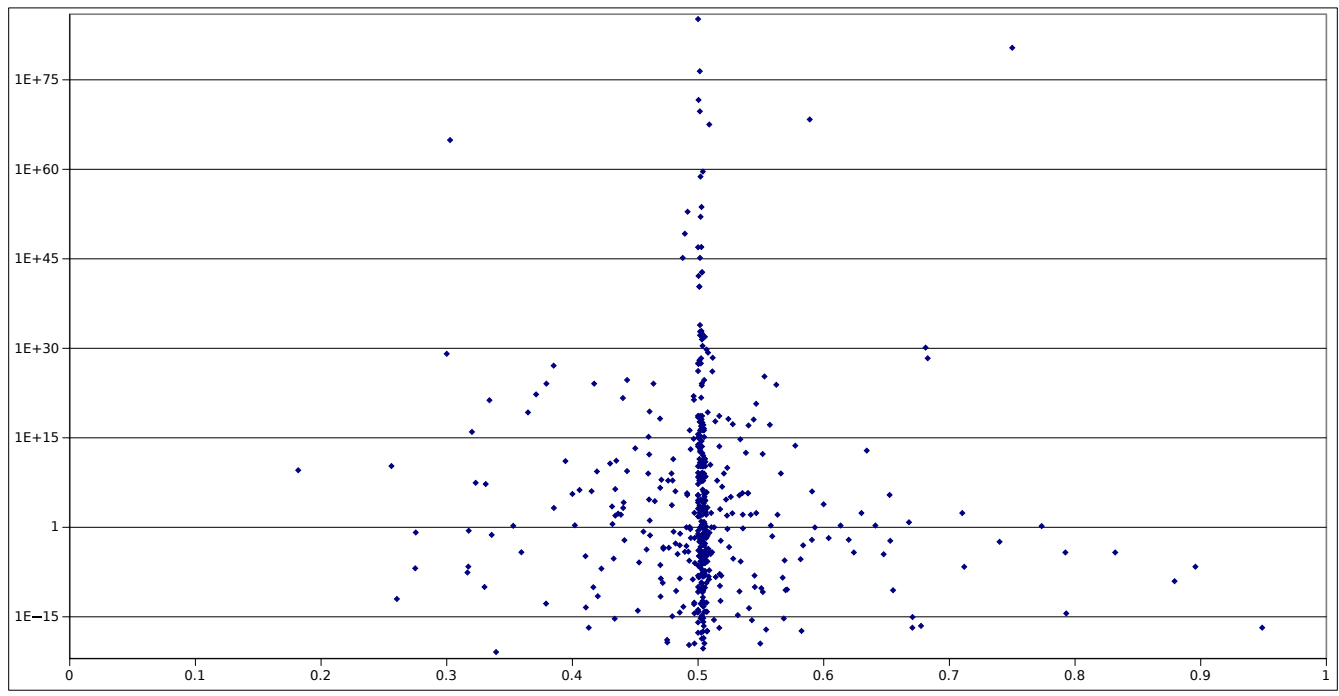

Рис. 1. Эффективность слияния выражений на словах длины $2^{20}(1 \mathrm{MiB})$

метод дает достаточно точные оценки для более широкого класса выражений, нежели $R F$ (на рисунке 1 для всевозможных пар выражений системы Snort на оси абсцисс отмечены относительные выигрыши в сокращении числа состояний, а на оси ординат - реальный относительный прирост числа слов длины $2^{20}$ при слиянии выражений). Так, среди всевозможных 528 пар выражений оценка осуществима на 239 парах выражений. Из них верхние оценки для 25 пар выражений лежат в промежутке от $10^{-2}$ до 1 , причем они отличаются в большую сторону от реальных значений для случая слов длины $2^{20}$ не более чем в 10 раз. Значения оценок для 187 пар не превосходят $10^{-2}$ и отличаются от реальных значений не более чем в 100 раз в большую сторону. 
Таким образом предложенный метод оценки, имеющий низкую вычислительную сложность, на практике позволяет с высокой точностью спрогнозировать относительный прирост числа слов при “слиянии" выражений не только из класса $R C \cap R F$, но и из более широкого подкласса класса $R C$.

\section{8. Заключение}

В статье рассмотрено расширение метода “слияния" регулярных выражений [11] для сокращения числа состояний детерминированных конечных автоматов, реализующих поиск по выражениям. Предложены оценки числа слов регулярных языков, задаваемых выражениями вида .* $\mathrm{R}_{1} * \mathrm{R}_{2} *$, а также метод оценки относительного прироста числа слов регулярных языков при "слиянии" пар выражений.

Автор выражает благодарность к.ф.-м.н. Галатенко Алексею Владимировичу и к.ф.-м.н. Панкратьеву Антону Евгеньевичу за постановку задачи и внимание к работе, а также брату Александрову Д. Е. за помощь в редактировании статьи.

\section{Список литературы}

1. Документация системы Snort, http://www.snort.org/documents.

2. Документаиия системь Bro, http://www.bro.org/.

3. Описание системы L7-filter, http://17-filter.sourceforge.net/README.

4. Странища аппаратных продуктов компании Cisco, http://www.cisco.com/c/en/us/ products/security/intrusion-prevention-system-ips.

5. Kumar S., Dharmapurikar S., Yu F., Crowley P., Turner J., "Algorithms to accelerate multiple regular expressions matching for deep packet inspection", ACM SIGCOMM Computer Communication Review, 36:4 (200), 339-350.

6. Aho A. V., Corasick M. J., "Efficient string matching: an aid to bibliographic search", Communications of the ACM, 18:6 (1975), 333-340.

7. Liu C., Wu J., "Fast Deep Packet Inspection with a Dual Finite Automata", Computers, IEEE Transactions on, 62:2 (2013), 310-321.

8. Kumar S., Chandrasekaran B., Turner J., Varghese G., "Curing regular expressions matching algorithms from insomnia, amnesia, and acalculia", Proc. 3rd ACM/IEEE Symp. on Architecture for networking and communications systems, ACM, 2007, 155-164.

9. Smith R., Estan C., Jha S., Kong S., "Deflating the big bang: fast and scalable deep packet inspection with extended finite automata", ACM SIGCOMM Computer Communication Review, 38:4 (2008), 207-218.

10. Yu F., Chen Z., Diao Y., Lakshman T. V., Katz R. H., "Fast and memory-efficient regular expression matching for deep packet inspection", Proc. of the 2006 ACM/IEEE symp. on Architecture for networking and communications systems, ACM, 2006, 93-102.

11. Александров Д. Е., "Об уменьшении автоматной сложности за счет расширения регулярных языков", Программная инженерия, 2014, № 11, 26-34.

12. Александров Д. Е., "Об оценках автоматной сложности распознавания класса регулярных языков”, Интеллектуалъные системы, 18:4 (2014), 121-146.

13. Документация регулярных выражений PCRE, http://www.pcre.org/pcre.txt.

14. Кудрявцев В. Б., Алешин С. В., Подколзин А. С., Введение в теорию автоматов, Наука, Москва, 1985.

15. Martin J. C., Introduction to Languages and the Theory of Computation, 4, McGraw-Hill, New York, 2011.

16. База сигнатур системы Snort, http://www.snort.org/snort-rules/. 